\title{
A unified electromechanical finite element dynamic analysis of multiple segmented smart plate energy harvesters - circuit connection patterns
}

\begin{abstract}
This paper presents the techniques for formulating the multiple segmented smart plate structures with different circuit connection patterns using the electromechanical finite element dynamic analysis. There are three major contributions in the proposed numerical studies. First, the electromechanical discretization has been developed for generalizing the coupled system of the Kirchhoff's smart plate structures with circuit connection patterns. Such constitutive numerical models reduced from the extended Lagrange equations can be used for the physical systems including, but not restricted to the multiple piezoelectric and electrode segments. Second, the multiple piezoelectric or electrode segments can be arranged electrically in parallel, series, and mixed series-parallel connections with the on-off switching techniques where the electrical outputs of each connection are further connected with the standard AC-DC circuit interfaces. Third, the coupling transformation technique (CTT) has been introduced by modifying the orthonormalized global element matrices into the scalar form equations. As a result, the multimode frequency response function and time waveform signal response equations are distinctly formulated for each circuit connection. Further parametric numerical case studies are also discussed in this paper. The benefit of using the circuit connection patterns with the on-off switching techniques is that the studies can be used for an adaptive vibration power harvester.
\end{abstract}

Keywords: Adaptive response · electromechanical discretization · energy harvesting · finite element methods $\cdot$ piezoelectric $\cdot$ smart structures $\cdot$ vibration.

\section{Introduction}

The vast majority of the electronic system demands of smart sensor wireless communication devices inevitably affect the electrical energy consumption. The system itself can be future microsystem devices for monitoring health conditions of industrial machines, infrastructures and even human activities. It is known that the use of the traditional power supply from batteries only gives limited energy during system operation resulting in the tedious and time consuming task of replacement. To alleviate such condition, self-charging and lifespan electrical energy required by the hybrid or integrated electronic systems can be obtained by converting the wasted or unused ambient vibrations of the surrounding environment into useful electrical energy using smart material systems. The investigations of piezoelectric components as smart material transducers have a profound effect on various engineering applications with mathematical modeling, thus making it an attractive field for researchers. Many researchers have been investigating the coupled piezoelectric and substructure systems (smart beams and plates) using finite element and analytical techniques in applications for active control systems [1]-[5], sensing and actuation systems [6][12] shape control system [13] and shunt circuit modeling [14]-[18].

Moreover, the development of piezoelectric vibration-based power harvesters has recently attracted researchers for investigating the engineering application of improving energysustainability. This includes the analytical models and experimental studies with the optimization

Mikail F Lumentut

School of Civil and Mechanical Engineering, Curtin University, Bentley, Perth, Australia

E-mail: m.lumentut@curtin.edu.au

Yi-Chung Shu

Institute of Applied Mechanics, National Taiwan University, Taiwan, Republic of China

E-mail: yichung@iam.ntu.edu.tw 
of designs from macro- to micro-electromechanical systems using mechanical and electrical circuit techniques. The various analytical studies of the piezoelectric power harvesting devices have been investigated using the lumped parameter models with electrical equivalent systems [19] and extensive circuit technique combinations [20]-[23], Rayleigh-Ritz methods [24]-[25] modal analysis method [26], assumed-mode method [27], and the weak-form techniques [28]-[29]. Other theoretical methods of piezoelectric power harvesting with different studies have also been investigated using random vibration analysis [30], fully closed-form boundary value methods [31][32], and analytical voltage- and charge-type Hamiltonian formulations [33]. For segmented piezoelectric structure, a few published works focusing on the structural discontinuity of piezoelectric components including beam orientation have investigated the use of the transfer matrix method [34] and analytical solutions [35]. Moreover, the effect of shunt control systembased the electrode distribution using the single piezoelectric beam structure for widening twofrequency bands and DC electrical output has been investigated using the charge-type analytical studies [36]. Most recently, the multiple segmented electrode configurations with shunt control system provided useful techniques for improving power harvesting with the multi-frequency and DC signal outputs [37]. Another trendy research effort for increasing the power harvester amplitudes for the wide ranging multi-frequency domain has also been considered as a new alternative solution by a few researchers. With the various analytical techniques, the technical formulations of the multiple piezoelectric bimorph beams connected using different electrical connection systems have been developed in [38]-[40]. Moreover, the use of impedance analytical [41] and SSHI approaches [42]-[43] have been rigorously developed. In recent research works using the analytical power harvesting studies, the smart plate structure with single and double piezoelectric segments connected with the AC and DC circuit connections have been developed in [44]-[45]. However, the system parameters of the piezoelectric mass and stiffness effects have been neglected. Then, the analytical model has been continuously developed by considering the piezoelectric mass and stiffness contributions [46]. However, the system was only based on the single piezoelectric segment with the power output across the load resistance.

The numerical studies of the various piezoelectric structures with the electrode effect have also been important techniques for developing the constitutive finite element equations in many different applications. The applied/generated voltage to/from the piezoelectric structures under dynamic excitation can occur if the distribution of the electrode layer on particular region of the piezoelectric is taken into account. This also depends upon the techniques for developing the equations. In some cases, many previous works in applications to the sensing and actuation and control systems have ignored the electrode effect. On the other hand, some numerical models have considered the electrode effects to the nodal [47]-[48] and elemental systems [3]-[4], [10], [49][50]. However, with the actual distributed electrode layer on the piezoelectric component, the combination between the multi-electric degree of freedoms and the mechanical degree of freedoms for formulating the global matrices have not yet been satisfied when formulating the frequency equations and DC signal analysis. For power harvesting application, the investigations of the electromechanical finite element analysis of piezoelectric power harvesters have been made by a few researchers. Modeling the finite element-based ANSYS multiphysics software has been incorporated with the SPICE software for the equivalent circuit model for simulating the power output [51]. Detail techniques of the system identification for the equivalent circuit parametersbased SSHI techniques has been presented using the COMSOL finite element simulation [43]. The finite element models with the electrode effect of the cantilevered beam [52] with different piezoelectric shapes and cantilevered plate [53] for the distributed piezoelectric layer have been presented. However, the electromechanical discretization for formulating orthonormalized global matrices and scalar forms have been ignored. The finite element model of the cantilevered piezoelectric bimorph beam structure with negligible electrode effects has also been investigated in [54]. Moreover, the numerical techniques in application to vibration energy harvester using the distributed piezoelectric beam with the electrode and damping effects were introduced into the derivations of coupled system [31], [55]. The technical concept underlies on formulating the set of the series multimode frequency response functions using the electromechanical discretization into the finite element equations.

In this paper, the smart plate structures with the multiple segment patterns, connected with the various circuit connection networks and standard AC-DC circuit interfaces, have been presented using the electromechanical finite element techniques. Only key numerical equations have been presented in this paper. The proposed electromechanical finite element vibration system can be highlighted in the following points: 
1. The electromechanical discretization (mechanical and electrical discretised elements) of the piezoelectric plate is introduced due to the physical system of the thin conducting electrode layer. It is an essential numerical technique underpinning the combination between the internal voltage and electromechanical system of the piezoelectric elements and the external circuit connection patterns.

2. The reduced constitutive electromechanical finite element equations can be used for the systems with the multiple piezoelectric or electrode segments that can be arranged electrically in parallel, series, and mixed series-parallel connections with the on-off switching techniques.

3. With the electrical connection patterns, the orthonormalized global element matrices can be transformed into the scalar form equations so that the FRFs and time waveform responses can be formulated.

4. The accuracy of the numerical studies can be obtained using the convergence criteria of the resonance frequencies corresponding with a number of electromechanical elements.

5. Detail parametric numerical studies are discussed using the option of multiple electrode segment connection systems. The multiple tier systems with the on-off switching techniques are utilized to the segment pattern connections. Each tier consists of the multiple electrode segments and each segment consists of the electromechanical discretized elements. It was found that increasing tier numbers using the parallel and mixed series-parallel electrode segment connections can increase the power amplitudes compared with the series electrode segment connection. However, when the ambient vibration environment is away from the resonance of the system, the mixed series-parallel electrode segment connection can be an effective option for widening the resonance frequency.

6. The strain mode shapes are utilized for analyzing the effect of power cancellations using each electrode connection patterns. The technique also provides of how to stimulate the power output under the strain node effects.

7. The DC time signal waveform analysis with the emphasis on the ripple signal effects from the rectifier and capacitor provides the information of the charging and discharging processes via the capacitor using different electrical loads.

8. The numerical techniques with the segment connection patterns and the on-off switched tier systems provide an effective tool to adaptively control the level of power output and frequency domain, particularly the system that depends on the ambient vibration environment. The studies can also be used for different applications (e.g., lightweight aerospace, automobile, and civil engineering structures) using the laminated smart plate structures with electrode layer.

\section{Coupled field constitutive equations}

Constitutive piezoelectric equations-based Kirchhoff's elemental plate can be formulated using the tensor electrical enthalpy concepts based on the continuum thermodynamics that can be condensed using Voigt's notation and Einstein's summation convention [56]-[59]. The electrical enthalpy for the smart material with Lead Zirconate Titanate (PZT) can be formulated under adiabatic and isothermal processes as,

$$
\hat{H}\left(S_{i}, E_{i}\right)=\frac{1}{2} \bar{C}_{11}^{E} S_{1}{ }^{2}+\bar{C}_{12}^{E} S_{1} S_{2}+\frac{1}{2} \bar{C}_{22}^{E} S_{2}{ }^{2}+\frac{1}{2} \bar{C}_{66}^{E} S_{6}{ }^{2}-\bar{e}_{31} E_{3} S_{1}-\bar{e}_{32} E_{3} S_{2}-\frac{1}{2} \bar{\varepsilon}_{33}^{S} E_{3}{ }^{2} .
$$

Here the general parameters $\bar{C}^{E}, \bar{e}, \bar{\varepsilon}^{S}, E, \boldsymbol{T}$, and $S$ represent the piezoelectric elastic stiffness at constant electric field, piezoelectric coefficient, permittivity under constant strain, electric field, stress, and strain, respectively. It is noted that the parameters of Eq. (1) were reduced from the plane-stress 3D into 2D components in order to obtain the correct parameter values of the Kirchhoff's elemental smart plate system. At this stage, Eq. (1) can be reformulated into matrix forms to give the following coupled field equations as,

$$
\begin{gathered}
\boldsymbol{T}_{p}=\left\{\begin{array}{l}
T_{1} \\
T_{2} \\
T_{6}
\end{array}\right\}=\left\{\begin{array}{l}
\partial \hat{H} / \partial S_{1} \\
\partial \hat{H} / \partial S_{2} \\
\partial \hat{H} / \partial S_{6}
\end{array}\right\}=\left[\begin{array}{ccc}
\bar{C}_{11}^{E} & \bar{C}_{12}^{E} & 0 \\
\bar{C}_{21}^{E} & \bar{C}_{22}^{E} & 0 \\
0 & 0 & \bar{C}_{66}^{E}
\end{array}\right]\left\{\begin{array}{l}
S_{1} \\
S_{2} \\
S_{6}
\end{array}\right\}-\left[\begin{array}{ccc}
0 & 0 & \bar{e}_{31} \\
0 & 0 & \bar{e}_{32} \\
0 & 0 & 0
\end{array}\right]\left\{\begin{array}{l}
E_{1} \\
E_{2} \\
E_{3}
\end{array}\right\}, \\
\boldsymbol{D}_{p}=\left\{\begin{array}{l}
D_{1} \\
D_{2} \\
D_{3}
\end{array}\right\}=\left\{\begin{array}{l}
-\partial \hat{H} / \partial E_{1} \\
-\partial \hat{H} / \partial E_{2} \\
-\partial \hat{H} / \partial E_{3}
\end{array}\right\}=\left[\begin{array}{ccc}
0 & 0 & 0 \\
0 & 0 & 0 \\
\bar{e}_{31} & \bar{e}_{32} & 0
\end{array}\right]\left\{\begin{array}{l}
S_{1} \\
S_{2} \\
S_{6}
\end{array}\right\}+\left[\begin{array}{ccc}
0 & 0 & 0 \\
0 & 0 & 0 \\
0 & 0 & \bar{\varepsilon}_{33}^{S}
\end{array}\right]\left\{\begin{array}{l}
E_{1} \\
E_{2} \\
E_{3}
\end{array}\right\},
\end{gathered}
$$


where $\bar{C}_{11}^{E}=C_{11}^{E}-\frac{C_{13}^{E^{2}}}{C_{33}^{E}}, \bar{C}_{12}^{E}=C_{12}^{E}-\frac{C_{13}^{E} C_{23}^{E}}{C_{33}^{E}}, \bar{C}_{21}^{E}=\bar{C}_{12}^{E}, \bar{C}_{22}^{E}=C_{22}^{E}-\frac{C_{23}^{E}}{C_{33}^{E}}, \bar{C}_{66}^{E}=C_{66}^{E}$,

$$
\bar{e}_{31}=e_{31}-\frac{C_{13}^{E} e_{33}}{C_{33}^{E}}, \quad \bar{e}_{32}=e_{32}-\frac{C_{23}^{E} e_{33}}{C_{33}^{E}}, \quad \bar{\varepsilon}_{33}^{S}=\varepsilon_{33}^{S}+\frac{e_{33}^{2}}{C_{33}^{E}} .
$$

The plane stress field from the Kirchhoff's isotropic plate substructure element and the general strain-displacement form [60]-[61] can be respectively formulated as,

$$
\boldsymbol{T}_{s}=\left\{\begin{array}{l}
T_{1} \\
T_{2} \\
T_{6}
\end{array}\right\}=\frac{E_{s}}{1-v^{2}}\left[\begin{array}{ccc}
1 & v & 0 \\
v & 1 & 0 \\
0 & 0 & \frac{1-v}{2}
\end{array}\right]\left\{\begin{array}{l}
S_{1} \\
S_{2} \\
S_{6}
\end{array}\right\}, \quad \boldsymbol{S}=\left\{\begin{array}{l}
S_{1} \\
S_{2} \\
S_{6}
\end{array}\right\}=-z\left\{\begin{array}{l}
\frac{\partial^{2} w}{\partial x^{2}} \\
\frac{\partial^{2} w}{\partial y^{2}} \\
2 \frac{\partial^{2} w}{\partial x \partial y}
\end{array}\right\} .
$$

Note that for the convenient purpose in the forthcoming notations, the superscripts 1 and 2 being used in the parameters of stress, strain, elastic stiffness, density, and volume represent the substructure and piezoelectric layers, respectively. It is obvious to see that Eqs. (2a)-(2b) and (3) can be stated, respectively as $\boldsymbol{T}^{(2)}=\boldsymbol{C}^{(2)} \boldsymbol{S}^{(2)}-\overline{\boldsymbol{e}}_{p}{ }^{T} \boldsymbol{E}$ and $\boldsymbol{D}=\overline{\boldsymbol{e}}_{p} \boldsymbol{S}^{(2)}+\overline{\boldsymbol{\varepsilon}}^{S} \boldsymbol{E}$ and $\boldsymbol{T}^{(1)}=\boldsymbol{C}^{(1)} \boldsymbol{S}^{(1)}$.

\section{Geometrical analysis of electromechanical plate element}

The physical smart plate structure under input base excitation as shown in Fig. 1a can be seen as an example of the actual system consisting of the piezoelectric, electrode, and substructure components. Also, example of the two coupled wires attached on the electrode layers includes on this part. In common practice, the piezoelectric material, available from the manufacturing company, covers with the thin electrode layer so that the electric voltage or current can be obtained during operating input base excitation. Fig. 1a also shows the arbitrary locations of the piezoelectric segments and electrode segments. As shown in Fig. 1b, the system shows different procedure when formulating the electromechanical finite element equations. It shows the electromechanical discretization consisting of mechanical and electrical discretized finite elements. The technical concept here was adopted from [55]. It can be seen in Fig. 1c that each equivalent electromechanical discretization on particular segment of piezoelectric or electrode component producing the single output of voltage consists of a number of certain elements that must be arranged electrically in the parallel connection. From there, the segment systems as given in Fig. $1 \mathrm{~d}$ can be further used for physical connection patterns such as parallel and series connections and even mixed series-parallel connections (detail discussions will be given in Section 4.2). One local element as shown in Fig. 1e is a given example for this case. The expression used in the solution form for four-node rectangular plate with the twelve unknown-degrees of freedom [62]-[64] is based on the non-conforming function that can be generally expressed as a polynomial function in the matrix form as,

where

$$
\begin{aligned}
& \boldsymbol{w}(x, y)=\boldsymbol{\eta}(x, y) \boldsymbol{c},
\end{aligned}
$$

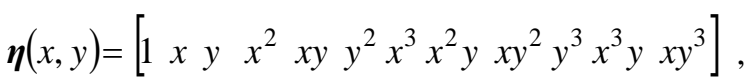

$$
\begin{aligned}
& \boldsymbol{c}=\left[\begin{array}{lllll}
c_{1} & c_{2} & c_{3} & \ldots \ldots . . c_{12}
\end{array}\right]^{T} \text {. }
\end{aligned}
$$

To specify the nodal vectors at each node for one element, the displacement vector fields can be extended into the twelve unknown nodal terms at four nodes $i, j, k$, and $l$ as,

$$
\begin{aligned}
& \boldsymbol{u}_{n}^{e}=\hat{\boldsymbol{\eta}}_{n}^{e} \boldsymbol{c} \quad \forall n \in\{i, j, k, l\}, \\
& \text { where } \quad \boldsymbol{u}_{n}^{e}=\left[\begin{array}{llll}
\boldsymbol{u}_{i} & \boldsymbol{u}_{j} & \boldsymbol{u}_{k} & \boldsymbol{u}_{l}
\end{array}\right]^{T}, \quad \hat{\boldsymbol{\eta}}_{n}^{e}=\left[\begin{array}{llll}
\boldsymbol{\eta}_{i} & \boldsymbol{\eta}_{j} & \boldsymbol{\eta}_{k} & \boldsymbol{\eta}_{l}
\end{array}\right]^{T}, \\
& \text { and } \quad \boldsymbol{u}_{i}=\left[w_{1} \theta_{x 1} \theta_{y 1}\right]^{T}, \boldsymbol{u}_{j}=\left[w_{2} \theta_{x 2} \theta_{y 2}\right]^{T}, \boldsymbol{u}_{k}=\left[w_{3} \theta_{x 3} \theta_{y 3}\right]^{T}, \boldsymbol{u}_{l}=\left[w_{4} \theta_{x 4} \theta_{y 4}\right]^{T} \text {, } \\
& \boldsymbol{\eta}_{n}=\left[\begin{array}{cccccccccccc}
1 & x_{n} & y_{n} & x_{n}{ }^{2} & x_{n} y_{n} & y_{n}{ }^{2} & x_{n}{ }^{3} & x_{n}{ }^{2} y_{n} & x_{n} y_{n}{ }^{2} & y_{n}{ }^{3} & x_{n}{ }^{3} y_{n} & x_{n} y_{n}{ }^{3} \\
0 & 0 & 1 & 0 & x_{n} & 2 y_{n} & 0 & x_{n}{ }^{2} & 2 x_{n} y_{n} & 3 y_{n}{ }^{2} & x_{n}{ }^{3} & 3 x_{n} y_{n}{ }^{2} \\
0 & -1 & 0 & -2 x_{n} & -y_{n} & 0 & -3 x_{n}{ }^{2} & -2 x_{n} y_{n} & -y_{n}{ }^{2} & 0 & -3 x_{n}{ }^{2} y_{n} & -y_{n}{ }^{3}
\end{array}\right] .
\end{aligned}
$$



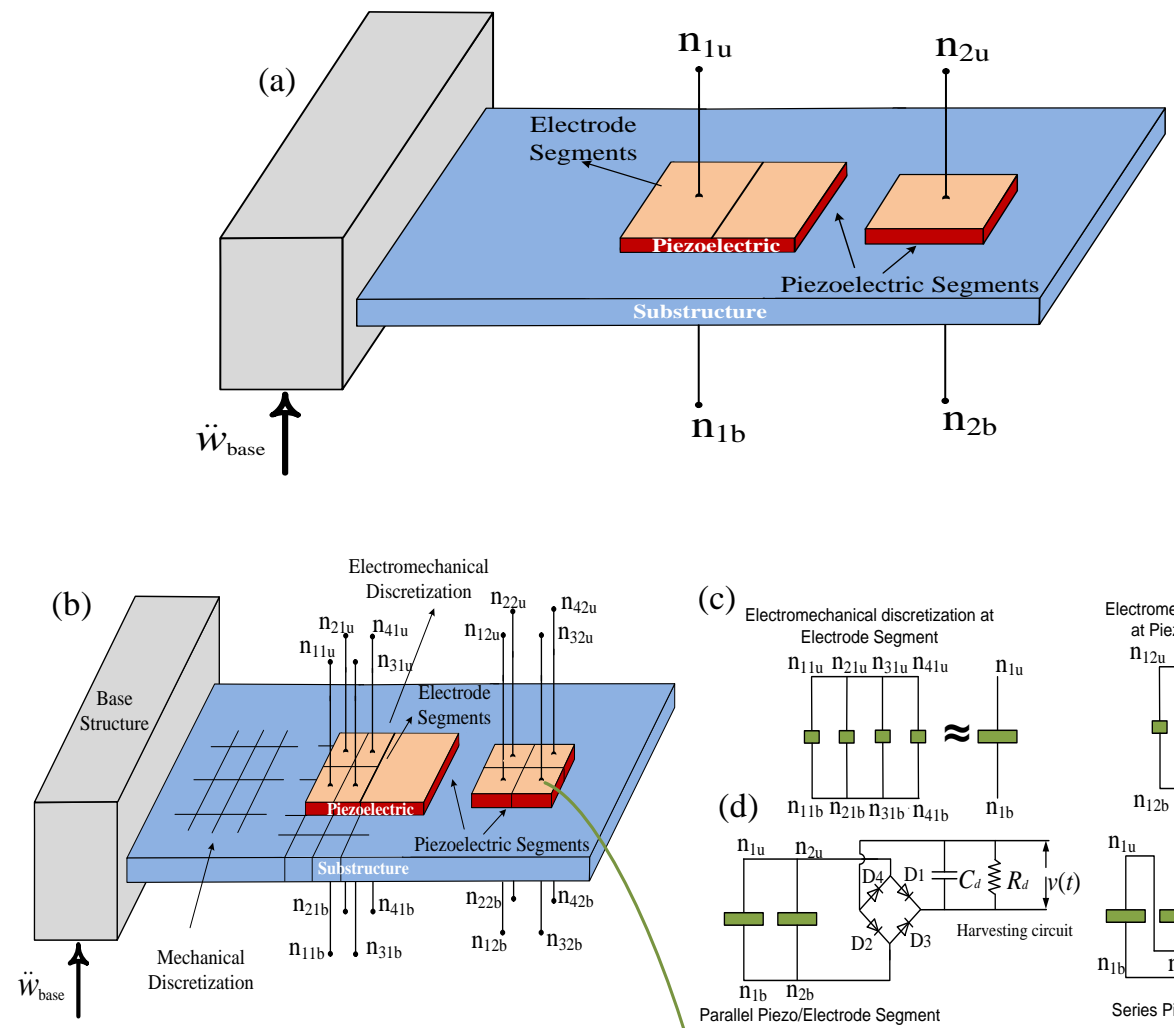

(c)
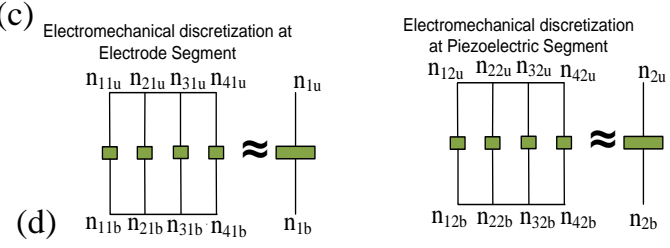

(d)
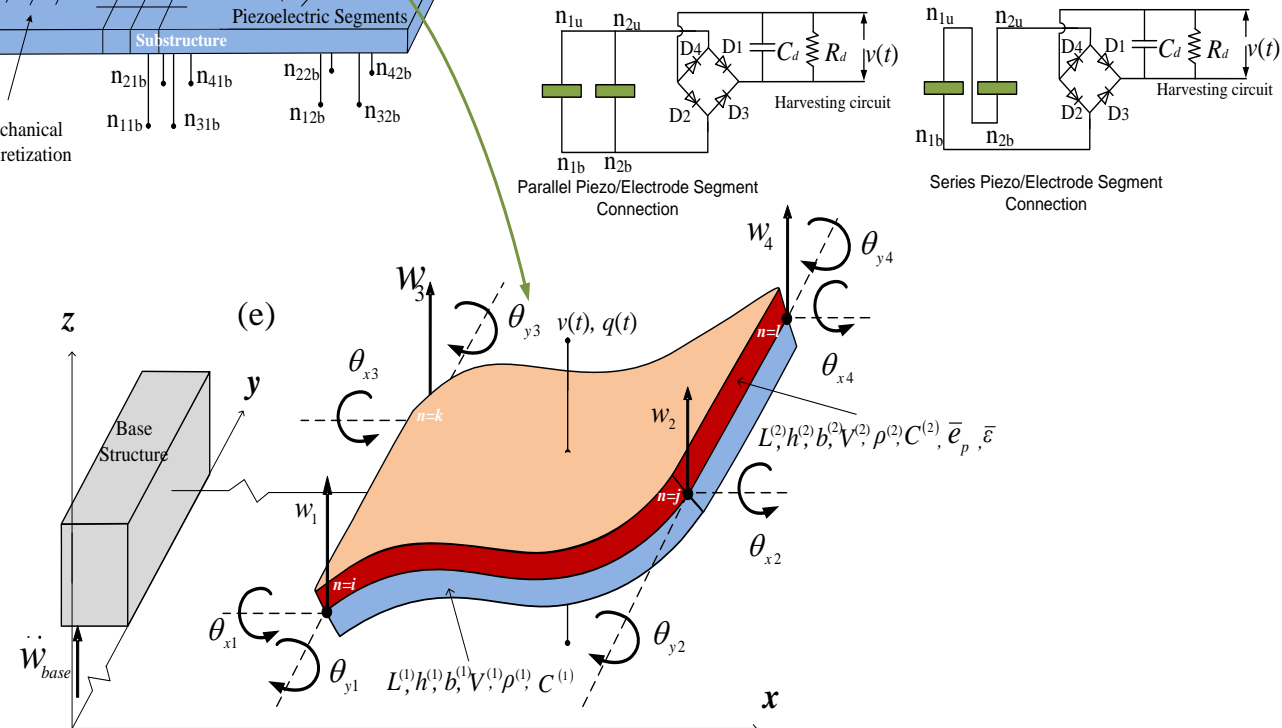

Fig.1 Smart plate structure modeling: (a) a physical or an actual system with the arbitrary electrode and piezoelectric segments, (b) electromechanical discretization, (c) equivalent electromechanical systems, (d) example of the circuit systems with standard AC-DC circuit interfaces, and (e) local element.

Note that since the general displacement function $\boldsymbol{w}_{n}$ must meet the continuity of the plate element, the general terms of the nodal vectors $\boldsymbol{\theta}_{x n}$ and $\boldsymbol{\theta}_{y n}$ correspond with the $\partial \boldsymbol{w}_{n} / \partial y$ and $-\partial w_{n} / \partial x$, respectively. The inverse of Eq. (5a) can be obtained using the twelve unknown parameters of the displacement field,

$$
\boldsymbol{c}=\hat{\boldsymbol{\eta}}_{n}^{e^{-1}} \boldsymbol{u}_{n}^{e} .
$$

For considering the smart plate system under dynamic motion, substituting Eq. (6) into Eq. (4a) gives,

$$
\boldsymbol{w}(x, y, t)=\boldsymbol{\Phi}_{n}^{e}(x, y) \boldsymbol{u}_{n}^{e}(t),
$$

where $\boldsymbol{\Phi}_{n}^{e}(x, y)=\boldsymbol{\eta}(x, y) \hat{\boldsymbol{\eta}}_{n}^{e^{-1}}$ is the shape function of size $1 \times 12$ for the displacement field of the non-conforming plate element. The strain-displacement field of the elemental plate from the second part of Eq. (3) can be reformulated using Eq. (7) to give, 
Eq. (8) can be reformulated as,

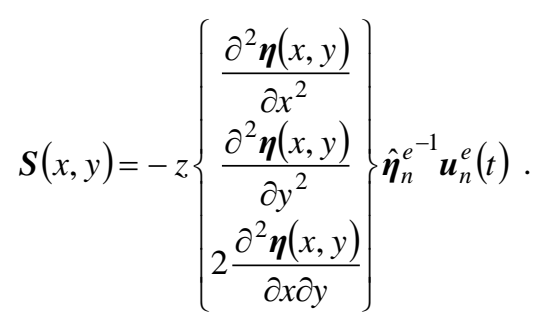

$$
\boldsymbol{S}(x, y)=-z \boldsymbol{B} \hat{\boldsymbol{\eta}}_{n}^{e^{-1}} \boldsymbol{u}_{n}^{e}(t)
$$

where $\boldsymbol{B}$ represents the matrix form of size $3 \times 12$ of the partial differential operator of polynomial terms,

$$
\boldsymbol{B}=\left[\begin{array}{cccccccccccc}
0 & 0 & 0 & 2 & 0 & 0 & 6 x & 2 y & 0 & 0 & 6 x y & 0 \\
0 & 0 & 0 & 0 & 0 & 2 & 0 & 0 & 2 x & 6 y & 0 & 6 x y \\
0 & 0 & 0 & 0 & 2 & 0 & 0 & 4 x & 4 y & 0 & 6 x^{2} & 6 y^{2}
\end{array}\right] .
$$

The elemental discretized electric field $\boldsymbol{E}$, induced by the strain field due to input ambient vibration, can create a polarization in the piezoelectric material in the $z$-direction along its thickness generating the electrical voltages. The electrical field can be formulated as,

$$
\boldsymbol{E}(x, y, z, t)=-\nabla \boldsymbol{\phi}=-\boldsymbol{\Omega}_{n}^{e}(x, y, z) \boldsymbol{v}^{e}(t),
$$

where $\phi=\vartheta \boldsymbol{v}^{e}(t)$ is the electrical potential and the parameter $\vartheta^{(e)}(z)=\left(z-z_{n}+h^{(2)}\right) / h^{(2)}$ is the shape function over the interval $z_{n}-h^{(2)} \leq z \leq z_{n}$ and asymmetric neutral axis $z_{n}=\frac{E_{p} h^{(1)^{2}}+E_{s} h^{(2)^{2}}+2 E_{p} h^{(1)} h^{(2)}}{2\left(E_{p} h^{(1)}+E_{s} h^{(1)}\right)}$. Like Eq. (3), Young's modulus of piezoelectric $E_{p}$ can also be obtained by further modifying Eq. (2c) because of having isotropic behavior. Note that only shape function in the thickness direction is considered due to the direction of electric field along the $z$ axis. Symbol $\nabla$ is a gradient operator for the first derivative of the shape function with respect to the thickness direction, giving $\boldsymbol{\Omega}_{n}^{e}(x, y, z)=\left[\begin{array}{lll}0 & 0 & \frac{\mathrm{d} \vartheta}{\mathrm{d} z}\end{array}\right]^{T}$. To obtain the expression of the stress field in the partial differential shape function form for both piezoelectric and plate elements, Eqs. (9) and (11) can be substituted into Eqs. (2a) and (3) to yield as,

$$
\begin{gathered}
\boldsymbol{T}^{(2)}=-z \boldsymbol{C}^{(2)} \boldsymbol{B} \hat{\boldsymbol{\eta}}_{n}^{e^{-1}} \boldsymbol{u}_{n}^{e}(t)+\overline{\boldsymbol{e}}_{p}{ }^{T} \boldsymbol{\Omega}^{e} \boldsymbol{v}^{e}(t), \\
\boldsymbol{T}^{(1)}=-z \boldsymbol{C}^{(1)} \boldsymbol{B} \hat{\boldsymbol{\eta}}_{n}^{e^{-1}} \boldsymbol{u}_{n}^{e}(t) .
\end{gathered}
$$

The electric displacement vector field can be further formulated using Eqs. (9), (11) and (2b) to give,

$$
\boldsymbol{D}=-z \overline{\boldsymbol{e}}_{p} \boldsymbol{B} \hat{\boldsymbol{\eta}}_{n}^{e^{-1}} \boldsymbol{u}_{n}^{e}(t)-\bar{\varepsilon} S \boldsymbol{\Omega}^{e} \boldsymbol{v}^{e}(t)
$$

To obtain the dynamic equations of the smart plate structure, all compatible equations can be solved using the extended Lagrange formulations as given in the next stage.

\section{Electromechanical finite element dynamic equations}

The extended Lagrange equations in terms of the electromechanical discretized finite element dynamic equations of the smart plate structure can be formulated as,

$$
\left.\begin{array}{l}
\frac{\mathrm{d}}{\mathrm{d} \mathrm{t}} \frac{\partial K E}{\partial \dot{\boldsymbol{u}}_{n}^{e}}+\frac{\partial P E}{\partial \boldsymbol{u}_{n}^{e}}-\frac{\partial W E}{\partial \boldsymbol{u}_{n}^{e}}-\frac{\partial W F}{\partial \boldsymbol{u}_{n}^{e}}=0 \\
\frac{\partial P E}{\partial \boldsymbol{v}^{\mathrm{e}}}-\frac{\partial W E}{\partial \boldsymbol{v}^{\mathrm{e}}}-\frac{\partial W F}{\partial \boldsymbol{v}^{\mathrm{e}}}=0
\end{array}\right\} \begin{gathered}
P E, W E \in\left\{\boldsymbol{u}_{n}^{e}, \boldsymbol{v}^{\mathrm{e}}\right\} \text { and } K E \in\left\{\dot{\boldsymbol{u}}_{n}^{e}\right\} \\
W F \in\left\{\boldsymbol{u}_{n}^{e}, \boldsymbol{v}^{\mathrm{e}}\right\}
\end{gathered} .
$$

It is important to note that since the plate structure is under moving base support, the reduced kinematic equations [55] can be used into this paper so as to formulate the functional energy forms. The relative displacement $\boldsymbol{w}(x, y, t)$ can be defined as the difference between absolute displacement $\boldsymbol{w}_{a b s}(x, y, t)$ and base excitation $\boldsymbol{w}_{\text {base }}(t)$. The kinetic energy for the elemental smart plate structure using Eq. (7) can be stated as, 


$$
\begin{aligned}
K E & =\frac{1}{2} \int_{V^{(1)}} \rho^{(1)} \dot{\boldsymbol{w}}(x, y, t)^{T} \dot{\boldsymbol{w}}(x, y, t) \mathrm{d} V^{(1)}+\frac{1}{2} \int_{V^{(2)}} \rho^{(2)} \dot{\boldsymbol{w}}(x, y, t)^{T} \dot{\boldsymbol{w}}(x, y, t) \mathrm{d} V^{(2)} \\
& =\frac{1}{2} \int_{V^{(1)}} \rho^{(1)} \dot{\boldsymbol{u}}_{n}^{e}(t)^{T} \hat{\boldsymbol{\eta}}_{n}^{e^{-T}} \boldsymbol{\eta}(x, y)^{T} \boldsymbol{\eta}(x, y) \hat{\boldsymbol{\eta}}_{n}^{e^{-1}} \dot{\boldsymbol{u}}_{n}^{e}(t) \mathrm{d} V^{(1)}+\frac{1}{2} \int_{V^{(2)}} \rho^{(2)} \dot{\boldsymbol{u}}_{n}^{e}(t)^{T} \hat{\boldsymbol{\eta}}_{n}^{e^{-T}} \boldsymbol{\eta}(x, y)^{T} \boldsymbol{\eta}(x, y) \hat{\boldsymbol{\eta}}_{n}^{e^{-1}} \dot{\boldsymbol{u}}_{n}^{e}(t) \mathrm{d} V^{(2)}
\end{aligned}
$$

The potential energy from the system can be written using Eqs. (9)-(10) and (12) as,

$$
\begin{aligned}
P E & =\frac{1}{2} \int_{V^{(1)}} \boldsymbol{S}^{(1)^{T}} \boldsymbol{T}^{(1)} \mathrm{d} V^{(1)}+\frac{1}{2} \int_{V^{(2)}} \boldsymbol{S}^{(2)^{T}} \boldsymbol{T}^{(2)} \mathrm{d} V^{(2)} \\
& =\frac{1}{2} \int_{V^{(1)}} z^{2} \boldsymbol{u}_{n}^{e}(t)^{T} \hat{\boldsymbol{\eta}}_{n}^{e^{-T}} \boldsymbol{B}^{T} \boldsymbol{C}^{(1)} \boldsymbol{B} \hat{\boldsymbol{\eta}}_{n}^{e^{-1}} \boldsymbol{u}_{n}^{e}(t) \mathrm{d} V^{(1)} \\
& +\frac{1}{2} \int_{V^{(2)}}\left(z^{2} \boldsymbol{u}_{n}^{e^{T}} \hat{\boldsymbol{\eta}}_{n}^{e^{-T}} \boldsymbol{B}^{T} \boldsymbol{C}^{(2)} \boldsymbol{B} \hat{\boldsymbol{\eta}}_{n}^{e^{-1}} \boldsymbol{u}_{n}^{e}(t)-z \boldsymbol{u}_{n}^{e^{T}} \hat{\boldsymbol{\eta}}_{n}^{e^{-T}} \boldsymbol{B}^{T} \overline{\boldsymbol{e}}_{p}^{T} \boldsymbol{\Omega}^{e} \boldsymbol{v}^{e}(t)\right) \mathrm{d} V^{(2)} .
\end{aligned}
$$

The electrical energy term for the piezoelectric element using Eqs. (11) and (13) can be formed as,

$$
\begin{aligned}
& W E=\frac{1}{2} \int_{V^{(2)}} \boldsymbol{E}^{T} \boldsymbol{D} \mathrm{d} V^{(2)}
\end{aligned}
$$

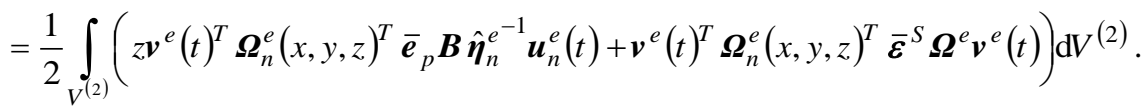

The non-conservative work done on the system due to the base excitation, input dynamic force and electrical charge output can be written as,

$$
\begin{aligned}
& W F=-\int_{V^{(1)}} \rho^{(1)} \boldsymbol{w}(x, y, t)^{T} \mathrm{~d} V^{(1)} \ddot{\boldsymbol{w}}_{\text {base }}(t)-\int_{V^{(2)}} \rho^{(2)} \boldsymbol{w}(x, y, t)^{T} \mathrm{~d} V^{(2)} \ddot{\boldsymbol{w}}_{\text {base }}(t)+\boldsymbol{u}_{n}^{e^{T}} \boldsymbol{F}_{c}+\boldsymbol{q}^{e}(t) \boldsymbol{v}^{e}(t) \\
& =-\int_{V^{(1)}} \rho^{(1)} \boldsymbol{u}_{n}^{e}(t)^{T} \hat{\boldsymbol{\eta}}_{n}^{e^{-T}} \boldsymbol{\eta}(x, y)^{T} \mathrm{~d} V^{(1)} \ddot{\boldsymbol{w}}_{\text {base }}(t)-\int_{V^{(2)}} \rho^{(2)} \boldsymbol{u}_{n}^{e}(t)^{T} \hat{\boldsymbol{\eta}}_{n}^{e^{-T}} \boldsymbol{\eta}(x, y)^{T} \mathrm{~d} V^{(2)} \ddot{\boldsymbol{w}}_{\text {base }}(t)+\boldsymbol{u}_{n}^{e^{T}} \boldsymbol{F}_{c}+\boldsymbol{q}^{e}(t) \boldsymbol{v}^{e}(t) .
\end{aligned}
$$

To include each expression from Eqs. (7), (9), (12)-(13), as required by Eqs. (15)-(18), the two electromechanical dynamic equations can be formulated separately using Eq. (14). After simplification, the local element matrices of the electromechanical dynamic equations by including Rayleigh damping can be further formulated as,

$$
\left[\begin{array}{cc}
\boldsymbol{M}^{e} & 0 \\
0 & 0
\end{array}\right]\left\{\begin{array}{l}
\ddot{\boldsymbol{u}}^{e}(t) \\
\ddot{\boldsymbol{v}}^{e}(t)
\end{array}\right\}+\left[\begin{array}{cc}
\boldsymbol{C}^{e} & 0 \\
\boldsymbol{P}_{\theta}^{e^{T}} & \boldsymbol{P}_{D}^{e}
\end{array}\right]\left\{\begin{array}{l}
\dot{\boldsymbol{u}}^{e}(t) \\
\dot{\boldsymbol{v}}^{e}(t)
\end{array}\right\}+\left[\begin{array}{cc}
\boldsymbol{K}^{e} & \boldsymbol{P}_{\theta}^{e} \\
0 & 0
\end{array}\right]\left\{\begin{array}{c}
\boldsymbol{u}^{e}(t) \\
\boldsymbol{v}^{e}(t)
\end{array}\right\}=\left\{\begin{array}{l}
\boldsymbol{F}^{e} \\
\boldsymbol{i}_{p}^{e}
\end{array}\right\},
$$

where

$$
\begin{aligned}
& \boldsymbol{M}^{e}=\sum_{n l=1}^{m} \hat{\boldsymbol{\eta}}_{(12 \times 12)}^{e^{-T}} \int_{V^{n l}} \rho^{(n l)} \underset{(12 \times 1)}{\boldsymbol{\eta}(x, y)^{T}} \underset{(1 \times 12)}{\boldsymbol{\eta}(x, y)} \mathrm{d} V^{(n l)} \underset{(12 \times 12)}{\hat{\boldsymbol{\eta}}_{n}^{e^{-1}}},
\end{aligned}
$$

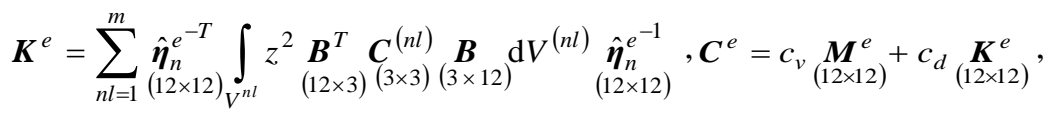

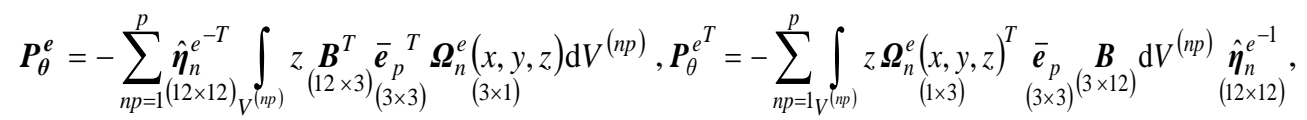

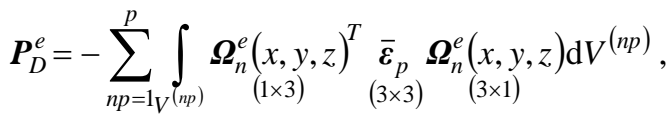

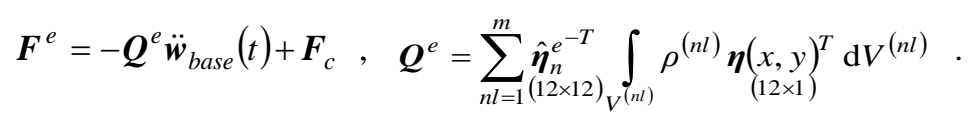

Note that Eq. (19a) includes input base excitation and dynamic force where next section will be further explained. The option of using one of them depends on the application. 


\subsection{Normalized global element matrices of electromechanical dynamic equation}

By scrutinizing the whole system consisting of the segmented piezoelectric/electrode components and the substructure, the electromechanical finite-element discretization for the smart structure must be computed and transformed from the local to global coordinate systems. The arrangement of the global element matrix of the smart plate structure can be formulated as,

$$
\begin{aligned}
& \underset{\left(n_{m} \times n_{m}\right)}{\boldsymbol{M}} \underset{\left(n_{m} \times 1\right)}{\ddot{\boldsymbol{u}}(t)}+\underset{\left(n_{m} \times n_{m}\right)}{\boldsymbol{C}} \underset{\left(n_{m} \times 1\right)}{\dot{\boldsymbol{u}}(t)}+\underset{\left(n_{m} \times n_{m}\right)}{\boldsymbol{K}} \underset{\left(n_{m} \times 1\right)}{\boldsymbol{u}(t)}+\underset{\left(n_{m} \times n_{e}\right)\left(n_{e} \times 1\right)}{\boldsymbol{P}_{\theta}} \underset{\left(n_{m} \times 1\right)}{\boldsymbol{v}(t)} \\
& \underset{\left(n_{e} \times n_{m}\right.}{\boldsymbol{P}_{(}^{T}} \underset{\left(n_{m} \times 1\right)}{\dot{\boldsymbol{u}}(\boldsymbol{t})}+\underset{\left(n_{e} \times n_{e}\right)}{\boldsymbol{P}_{D}} \underset{\left(n_{e} \times 1\right)}{\dot{\boldsymbol{v}}(\boldsymbol{t})}=\underset{\left(\begin{array}{c}
\left.n_{e} \times 1\right) \\
n_{e}(t)
\end{array}\right)}{\boldsymbol{i}}
\end{aligned}
$$

It is noted that the matrix sizes of each component of Eq. (20) correspond with mechanical degrees of freedom for each node $n_{m}$ and electrical degrees of freedom for each element $n_{e}$.

The solution form of time-dependent displacement can be stated in terms of the normalized modal vector and generalized coordinate as,

$$
\boldsymbol{u}(t)=\boldsymbol{\varphi}_{1} \boldsymbol{a}_{1}(t)+\boldsymbol{\varphi}_{2} \boldsymbol{a}_{2}(t)+\ldots+\boldsymbol{\varphi}_{m-1} \boldsymbol{a}_{m-1}(t)+\boldsymbol{\varphi}_{m} \boldsymbol{a}_{m}(t)=\boldsymbol{\varphi} \boldsymbol{a}(t) .
$$

where parameter $\boldsymbol{\varphi}=\left[\begin{array}{llll}\boldsymbol{\varphi}_{1} & \boldsymbol{\varphi}_{2} & \ldots & \boldsymbol{\varphi}_{m}\end{array}\right]$ is a normalized modal matrix that must meet the orthonormality relation with $\boldsymbol{\varphi}^{\mathrm{T}} \boldsymbol{M} \boldsymbol{\varphi}=1$. Note that although the expression given in Eq. (21) shows a similar form to that of given in [31], the smart plate structure shows more complex physical system compared with the smart beam structure giving different normalized modal matrix with increasing degrees of freedom. At this point, the normalized eigenvector or modal matrix can simply be formulated as $\boldsymbol{\varphi}=\boldsymbol{U} /\left(\boldsymbol{U}^{\mathrm{T}} \boldsymbol{M} \boldsymbol{U}\right)^{1 / 2}$. The parameter $\boldsymbol{U}=\left[\begin{array}{llll}\boldsymbol{U}_{1} & \boldsymbol{U}_{2} & \ldots & \boldsymbol{U}_{m}\end{array}\right]$ is the known value of each eigenvector for each particular degree of freedom or eigenvalue that can be obtained from expression $\left[\chi-\omega^{2} \boldsymbol{I}\right] \boldsymbol{U}=0$ where $\boldsymbol{I}=\boldsymbol{M}^{-1} \boldsymbol{M}, \boldsymbol{\chi}=\boldsymbol{M}^{-1} \boldsymbol{K}$.

Normalized global matrix equations can be formulated by substituting Eq. (21) into Eqs. (20a)(20b) and premultiplying the result by $\varphi^{T}$ and the result of which can simply be formulated as,

$$
\begin{gathered}
\ddot{\boldsymbol{a}}(t)+2 \zeta \omega \dot{\boldsymbol{a}}(t)+\omega^{2} \boldsymbol{a}(t)+\hat{\boldsymbol{P}}_{\boldsymbol{\theta}} \boldsymbol{v}(t)=-\hat{\boldsymbol{Q}} \ddot{\boldsymbol{w}}_{\text {base }}(t)+\hat{\boldsymbol{F}}_{c}, \\
\hat{\boldsymbol{P}}_{\theta}^{T} \dot{\boldsymbol{a}}(\mathrm{t})+\boldsymbol{P}_{\mathrm{D}} \dot{\boldsymbol{v}}(\mathrm{t})=\boldsymbol{i}_{\boldsymbol{p}}(\mathrm{t}) .
\end{gathered}
$$

where orthonormalized parameters from Eq. (22) can be stated as,

$$
\begin{gathered}
\boldsymbol{\varphi}^{\boldsymbol{T}} \boldsymbol{M} \boldsymbol{\varphi}=\boldsymbol{I}, \quad \boldsymbol{\varphi}^{\boldsymbol{T}} \boldsymbol{K} \boldsymbol{\varphi}=\operatorname{diag}\left(\omega_{1}{ }^{2}, \omega_{2}{ }^{2}, \cdots, \omega_{r}{ }^{2}\right), \\
\boldsymbol{\varphi}^{\boldsymbol{T}} \boldsymbol{C} \boldsymbol{\varphi}=c_{v}\left(\boldsymbol{\varphi}^{\mathrm{T}} \boldsymbol{M} \boldsymbol{\varphi}\right)+c_{d}\left(\boldsymbol{\varphi}^{\mathrm{T}} \boldsymbol{K} \boldsymbol{\varphi}\right)=c_{v} \boldsymbol{I}+c_{d} \omega^{2}=2 \zeta \omega \\
\hat{\boldsymbol{P}}_{\boldsymbol{\theta}}=\boldsymbol{\varphi}^{T} \boldsymbol{P}_{\theta}, \quad \hat{\boldsymbol{P}}_{\theta}^{T}=\boldsymbol{P}_{\theta}^{T} \boldsymbol{\varphi}, \quad \hat{\boldsymbol{Q}}=\boldsymbol{\varphi}^{T} \boldsymbol{Q}, \hat{\boldsymbol{F}}_{c}=\boldsymbol{\varphi}^{T} \boldsymbol{F}_{c} .
\end{gathered}
$$

Note that Eq. (22a) shows two input dynamic motions where it show general equation. However, the option of using one of the input terms into the computational process depends on the application. In the next section, further formulations will be based on the input base excitation where the input dynamic force will be given separately to the equations as an example.

4.2 Coupling transformation techniques of the normalized global element matrices into the scalar form equations with various circuit connections

In this section, the use of multiple segment patterns of the smart plate structure system can be formulated using the CTT. It should be noted that since all parameters in Eq. (20) provide large and different matrix sizes, direct operation for such equation may lead to a challenging computational process. In particular, the smart plate structure is coupled with the complex circuit connection patterns and AC-DC rectification. To alleviate such conditions, the CTT with the orthonormality for generalizing electromechanical dynamic equations has been introduced by modifying the matrices of Eq. (20) into the scalar form equations in order to give the effective and efficient computational processes. As shown in the next stage, the iterative series FRF equations will show distinct formulas for each circuit segment connection. As mentioned previously, the equations can be used for the physical system of either the multiple piezoelectric or electrode segments. To avoid confusion with these two terms, the system with the multiple piezoelectric segments refers to the segments of both piezoelectric and electrode components. The reason is that 
the surface of the piezoelectric material, inherently produced by the manufacturing company, covers with the thin conducting electrode layer so as to produce the voltage signal.
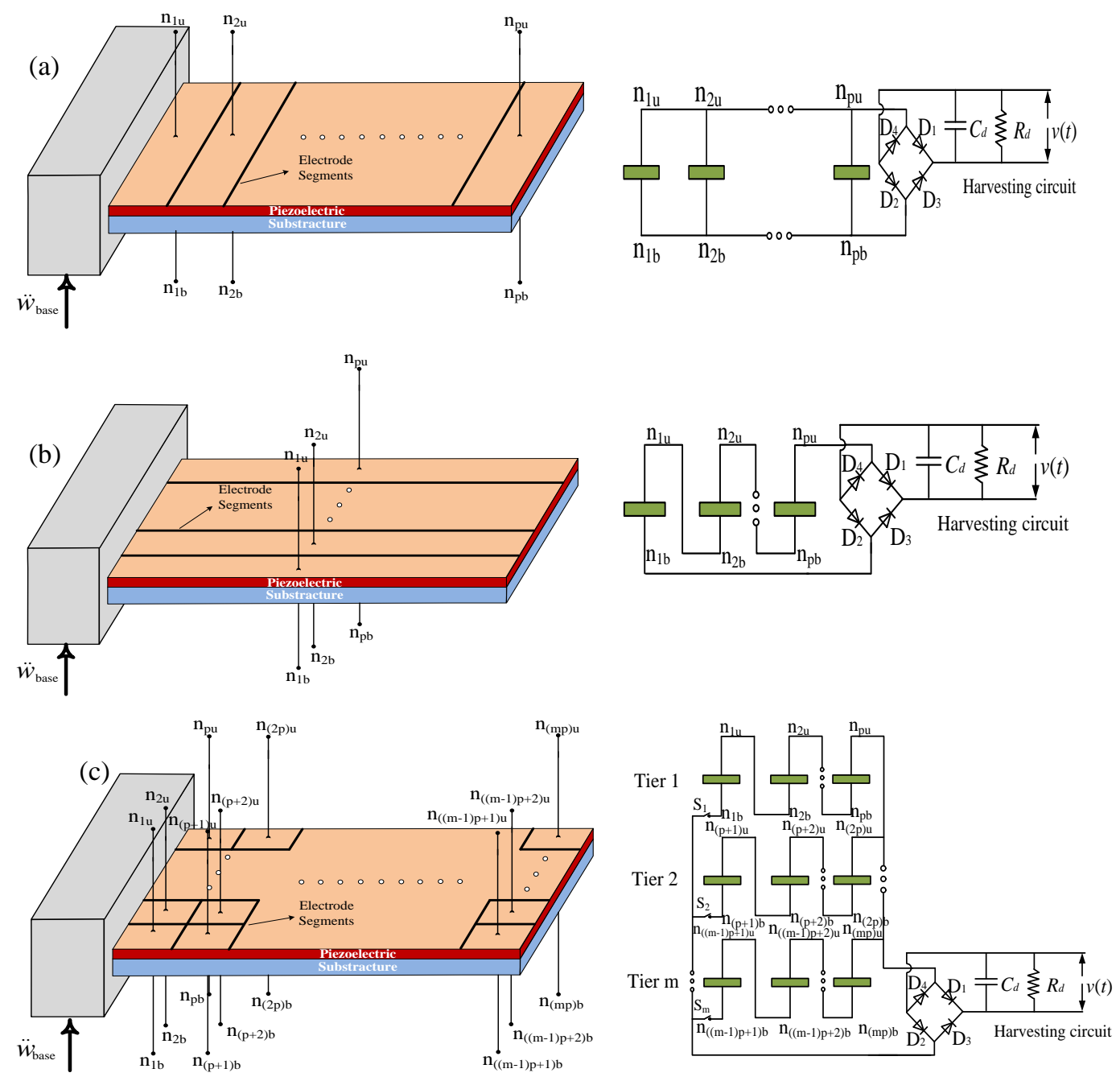

Fig. 2 Smart plate structure modeling with equivalent electromechanical systems and standard AC-DC circuit interfaces: (a) the parallel electrode segment connections, (b) the series electrode segment connection, and (c) the mixed series-parallel electrode segment connection with the on-off switching technique.

Thus, when the piezoelectric material is cut into the segments of the particular shape, the electrode on it is also affected. However, the system with the multiple electrode segments refers to the distributed piezoelectric structure covered with the segmented electrodes. This can occur when scoring or etching the electrode layer from the piezoelectric material. The piezoelectric/electrode segments can be arranged using the electrical parallel, series and mixed series-parallel connections as shown in Fig. 2 where their formulations are given in the forthcoming sections. It is important to note here that the segment patterns shown in Fig. 2 are only example for simplicity in order to reduce the constitutive frequency equations and time signal waveform equations. Arbitrary segments can also be used using the same equations. For conveniently modeling the electromechanical finite-element discretization system, the normalized global matrices can be transformed into the scalar form equations. The MATLAB program computation was developed for modeling these techniques. The essential parts of computational method provide not only the generalized mechanical components (mass, stiffness and damping matrices), but also most importantly the generalized internal capacitances and normalized piezoelectric couplings for each element corresponding to the mechanical multi degree of freedom for the whole discretized system structure. In this case, the first form of the generalized electromechanical dynamic equations can be formulated in the mechanical multimode system $r=1,2,3, \ldots, N D O F$ in terms of the number of normalized piezoelectric elements for the first segment $s_{1}=1_{1}, 2_{1}, 3_{1}, \ldots, N E L P_{1}$, the second segment $s_{2}=1_{2}, 2_{2}, 3_{2}, \ldots ., N E L P_{2}$ and $p^{\text {th }}$ segment $s_{p}=1_{p}, 2_{p}, 3_{p}, \ldots ., N E L P_{p}$ as, 


$$
\begin{gathered}
\ddot{a}_{1}(t)+2 \zeta_{1} \omega_{1} \dot{a}_{1}(t)+\omega_{1}{ }^{2} a_{1}(t)+\hat{P}_{11_{1}} v_{1_{1}}(t)+\hat{P}_{12_{1}} v_{2_{1}}(t)+\cdots+\hat{P}_{1 s_{1}} v_{s_{1}}(t) \\
+\hat{P}_{11_{2}} v_{1_{2}}(t)+\hat{P}_{12_{2}} v_{2_{2}}(t)+\cdots+\hat{P}_{1 s_{2}} v_{s_{2}}(t) \\
\ldots+\hat{P}_{11_{p}} v_{1_{p}}(t)+\hat{P}_{12_{p}} v_{2_{p}}(t)+\cdots+\hat{P}_{1 s_{p}} v_{s_{p}}(t)=-\hat{Q}_{1} \ddot{w}_{\text {base }}(t) \\
\ddot{a}_{2}(t)+2 \zeta_{2} \omega_{2} \dot{a}_{2}(t)+\omega_{2}{ }^{2} a_{2}(t)+\hat{P}_{21_{1}} v_{1_{1}}(t)+\hat{P}_{22_{1}} v_{2_{1}}(t)+\cdots+\hat{P}_{2 s_{1}} v_{s_{1}}(t) \\
+\hat{P}_{21_{2}} v_{1_{2}}(t)+\hat{P}_{22_{2}} v_{2_{2}}(t)+\cdots+\hat{P}_{2 s_{2}} v_{s_{2}}(t) \\
\ldots+\hat{P}_{21_{p}} v_{1_{p}}(t)+\hat{P}_{22_{p}} v_{2_{p}}(t)+\cdot \cdots+\hat{P}_{2 s_{p}} v_{s_{p}}(t)=-\hat{Q}_{2} \ddot{w}_{\text {base }}(t), \\
\cdot \\
\cdot \\
\ddot{a}_{r}(t)+2 \zeta_{r} \omega_{r} \dot{a}_{r}(t)+\omega_{r}{ }^{2} a_{r}(t)+\sum_{s_{1}=1_{1}}^{N E L P_{1}} \hat{P}_{r s_{1}} v_{s_{1}}(t)+\sum_{s_{2}=1_{2}}^{N E L P_{2}} \hat{P}_{r s_{2}} v_{s_{2}}(t)+\cdots+\sum_{s_{p}=1_{p}}^{N E L P_{p}} \hat{P}_{r s_{p}} v_{s_{p}}(t)=-\hat{Q}_{r} \ddot{w}_{\text {base }}(t), \\
r=1,2, \ldots, N D O F .
\end{gathered}
$$

The second electromechanical dynamic equation relating to the electrical current output due to the effect of the first piezoelectric/electrode segment can be formulated as,

$$
\begin{gathered}
\hat{P}_{1_{1}} \dot{a}_{1}(t)+\hat{P}_{1_{1}} \dot{a}_{2}(t)+\cdots+\hat{P}_{s_{1} r} \dot{a}_{r}(t)+P_{D 1_{1}} \dot{v}_{1_{1}}(t)=i_{P 1_{1}}(t), \\
\hat{P}_{2_{1}} \dot{a}_{1}(t)+\hat{P}_{2_{1}} \dot{a}_{2}(t)+\cdots+\hat{P}_{s_{1} r} \dot{a}_{r}(t)+P_{D 2_{1}} \dot{v}_{2_{1}}(t)=i_{P 2_{1}}(t), \\
\cdot \\
\sum_{r=1}^{N D O F} \hat{P}_{s_{1}} \dot{a}_{r}(t)+P_{D s_{1}} \dot{v}_{s_{1}}(t)=i_{P_{s_{1}}}(t), \quad s_{1}=1_{1}, 2_{1}, 3_{1}, \ldots, N E L P_{1} .
\end{gathered}
$$

For the second piezoelectric/electrode segment, the second electromechanical dynamic equation can be formulated as,

$$
\begin{gathered}
\hat{P}_{1_{2}} \dot{a}_{1}(t)+\hat{P}_{1_{2}} \dot{a}_{2}(t)+\cdots+\hat{P}_{s_{2} r} \dot{a}_{r}(t)+P_{D 1_{2}} \dot{v}_{1_{2}}(t)=i_{P 1_{2}}(t), \\
\hat{P}_{2_{2}} \dot{a}_{1}(t)+\hat{P}_{2_{2} 2} \dot{a}_{2}(t)+\cdots+\hat{P}_{s_{2} r} \dot{a}_{r}(t)+P_{D 2_{2}} \dot{v}_{2_{2}}(t)=i_{P 2_{2}}(t), \\
\cdot \\
\cdot \\
\sum_{r=1}^{N D O F} \hat{P}_{s_{2} r} \dot{a}_{r}(t)+P_{D s_{2}} \dot{v}_{s_{2}}(t)=i_{P_{s_{2}}}(t), \quad s_{2}=1_{2}, 2_{2}, 3_{2}, \ldots ., N E L P_{2} .
\end{gathered}
$$

For multi-piezoelectric/electrode segments, the second electromechanical dynamic equation can be formulated as,

$$
\begin{gathered}
\hat{P}_{1_{1}} \dot{a}_{1}(t)+\hat{P}_{1_{p} 2} \dot{a}_{2}(t)+\cdots+\hat{P}_{s_{p} r} \dot{a}_{r}(t)+P_{D 1_{p}} \dot{v}_{1_{p}}(t)=i_{P 1_{p}}(t), \\
\hat{P}_{2_{p} 1} \dot{a}_{1}(t)+\hat{P}_{2_{p} 2} \dot{a}_{2}(t)+\cdots+\hat{P}_{s_{p}} \dot{a}_{r}(t)+P_{D 2_{p}} \dot{v}_{2_{p}}(t)=i_{P 2_{p}}(t), \\
\cdot \\
\cdot \\
\sum_{r=1}^{N D O F} \hat{P}_{s_{p} r} \dot{a}_{r}(t)+P_{D s_{p}} \dot{v}_{s_{p}}(t)=i_{P_{p}}(t), \quad s_{p}=1_{p}, 2_{p}, 3_{p}, \ldots ., N E L P_{p} .
\end{gathered}
$$

It is important to note here that since the electrical segmented piezoelectric/electrode systems are formulated in Eqs. (24)-(27), the electrical discretized elements for each segment must be generalized into the internal parallel connection due to the distributed electrode onto surface of the piezoelectric structure as,

$$
\begin{aligned}
& \overbrace{v_{1_{1}}(t)=v_{2_{1}}(t)=\cdots=v_{s_{1}}(t)}^{\text {Piezo / Electrode Segment } 1}, \overbrace{v_{1_{2}}(t)=v_{2_{2}}(t)=\cdots=v_{S_{2}}(t)}^{\text {Piezol Electrode Segment } 2}, \overbrace{v_{1_{p}}(t)=v_{2_{p}}(t)=\cdots=v_{s_{p}}(t)}^{\text {Piezo/ Electrode Segment } p}, \\
& \overbrace{i_{P 1_{1}}(t)+i_{P 2_{1}}(t)+\cdots+i_{P_{N E L L_{1}}}(t)=i_{P_{S_{1}}}(t)}^{\text {Piezo/ Electrode Segment } 1}, \overbrace{i_{P 1_{2}}(t)+i_{P 2_{2}}(t)+\cdots+i_{P_{N E L P_{2}}}(t)=i_{P s_{2}}(t)}^{\text {Piectrode Segment } 2},
\end{aligned}
$$




$$
\overbrace{i_{P 1_{p}}(t)+i_{P 2_{p}}(t)+\cdots+i_{P_{N E L P_{p}}}(t)=i_{P s_{p}}(t)}^{\text {Piezo/ Electrode Segment } p} .
$$

Equations (25)-(27) can be reformulated after applying Eqs. (28b)-(28c) to give,

$$
\begin{gathered}
\sum_{r=1}^{N D O F} \sum_{s_{1}=1_{1}}^{N E L P} \hat{P}_{s_{1}} \dot{a}_{r}(t)+\sum_{s_{1}=1}^{N E L P} P_{D s_{1}} \dot{v}_{s_{1}}(t)=i_{P s_{1}}(t), \\
\sum_{r=1}^{N D O F} \sum_{s_{2}=1_{2}}^{N E L P} \hat{P}_{s_{2}} \dot{r}_{r}(t)+\sum_{s_{2}=1_{2}}^{N E L P} P_{D s_{2}} \dot{v}_{s_{2}}(t)=i_{P_{s_{2}}}(t), \\
\cdot \\
\sum_{r=1}^{N D O F} \sum_{s_{P}=1_{P}}^{N E L P} \hat{P}_{s_{p}} \dot{a}_{r}(t)+\sum_{s_{P}=1}^{N} P_{D s_{p}} \dot{v}_{s_{p}}(t)=i_{P_{s_{p}}}(t) .
\end{gathered}
$$

As discussed previously, the multi-piezoelectric/electrode segments can be electrically connected using the parallel, series, and mixed parallel-series connections where the $R C$ harvesting circuit can be used to connect all networks of each piezoelectric/electrode segment connection. Here, the external parallel connection can be formulated as,

$$
\begin{aligned}
& \overbrace{v_{s_{1}}(t)}^{\text {Piezo/ Electrode Segment } 1}=\overbrace{v_{s_{2}}(t)}^{\text {Piezo/ElectrodeSegment } 2}=\cdots=\overbrace{v_{s_{p}}(t)}^{\text {Piezo/ElectrodeSegment }} p=v(t), \\
& \text { Piezo/ElectrodeSegment } 1 \text { Piezo/Electrode Segment } 2 \text { Piezo/Electrode Segment } p \\
& \overbrace{i_{P s_{1}}(t)}+\overbrace{i_{P s_{2}}(t)}+\cdots+\overbrace{i_{P s_{p}}(t)}=i_{P}(t) .
\end{aligned}
$$

The choice of external series connection for multi-piezoelectric/electrode segment pattern can also be conducted and formulated as,

$$
\begin{aligned}
& \text { Piezo/ElectrodeSegment } 1 \text { Piezo/ElectrodeSegment } 2 \text { Piezo/ElectrodeSegment } p \\
& \overbrace{v_{s_{1}}(t)}+\overbrace{v_{s_{2}}(t)}+\cdots+\overbrace{v_{s_{p}}(t)}=v(t), \\
& \text { Piezo/ElectrodeSegment } 1 \text { Piezo/Electrode Segment } 2 \text { Piezo/Electrode Segment } p \\
& \overbrace{i_{P_{s_{1}}}(t)}=\overbrace{i_{P_{S_{2}}}(t)}=\cdots=\overbrace{i_{P_{S_{p}}}(t)}=i_{P}(t) .
\end{aligned}
$$

The mixed series-parallel connection for piezoelectric/electrode segments can be arranged into the multiple tier array. Each tier, arranged into the parallel connection, consists of the series piezoelectric/electrode segment array, giving different voltage with the same current. In Fig. 2c, each tier can be controlled using the on-off switching technique. In terms of the KVL system, the series connection pattern of multi-piezoelectric/electrode segment for each tier can be formulated as,

$$
\begin{aligned}
& \overbrace{v_{s_{1}}^{\text {tier } 1}(t)}^{\text {Piezo/ Electrode Segment } 1}+\overbrace{v_{s_{2}}^{\text {tier } 1}(t)}^{\text {Piezo/ Electrode Segment } 2}+\cdots+\overbrace{v_{s_{p}}^{\text {tier } 1}(t)}^{\text {Piezo/ Electrode Segment }} p=v^{\text {tier } 1}(t), \\
& \text { Piezo/Electrode Segment }(p+1) \quad \text { Piezo/ Electrode Segment }(p+2) \quad \text { Piezo/ Electrode Segment } 2 p
\end{aligned}
$$

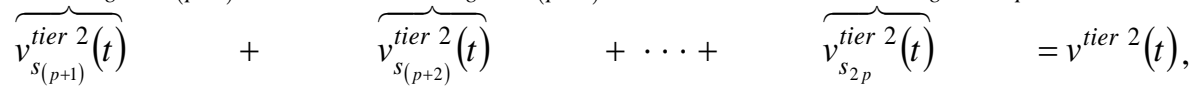

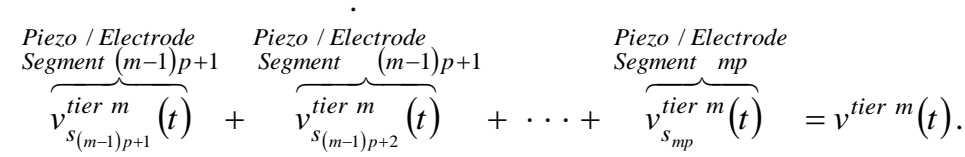

Note that since the total voltage of each tier must be equal, the current through each tier must be added to give the current output across the external circuit. At this case, the parallel connection pattern of $m^{\text {th }}$ tier can be formulated using the KCL system as,

$$
\begin{gathered}
v^{\text {tier } 1}(t)=v^{\text {tier } 2}(t)=\cdots=v^{\text {tier } m}(t)=v(t), \\
i_{P s_{p}}^{\text {tier } 1}(t)+i_{P s_{2 p}}^{\text {tier } 2}(t)+\cdots+i_{P s_{m p}}^{\text {tier } m}(t)=i_{p}(t) .
\end{gathered}
$$


For the RC harvesting circuit, the circuit equation can be formulated as,

$$
i_{P}(t)=C_{d} \dot{v}(t)+\frac{v(t)}{R_{d}} .
$$

Note that Eq. (34) can be used for connecting the segment connection systems.

\subsection{The solution form of parallel piezoelectric/electrode segment connections}

To formulate the multimode frequency response functions of the parallel electrical segmented piezoelectric/electrode system as shown in Fig. 2a, Eqs. (24a)-(24c) and (29a)-(29c) associated with Eqs. (30a)-(30b) and (34) can be solved simultaneously using Laplace transform giving the superposition of equations in matrix form. In this stage, after manipulation and simplification, the electrical voltage multimode FRF for multiple piezoelectric/electrode segments can be formulated as,

$$
\frac{v(j \omega)}{-\omega^{2} w_{\text {base }} e^{j \omega t}}=\frac{\sum_{r=1}^{N D O F} \frac{j \omega B_{s r} \hat{Q}_{r}}{A_{r}}}{\left(j \omega D_{s}-C-\sum_{r=1}^{N D O F} \frac{j \omega B_{r s} B_{s r}}{A_{r}}\right)},
$$

where

$$
A_{r}=\omega_{r}^{2}-\omega^{2}+j 2 \zeta_{r} \omega_{r} \omega, \quad C=j \omega C_{d}+\frac{1}{R_{d}}
$$

$$
\begin{gathered}
B_{r s}=\sum_{s_{1}=1_{1}}^{N E L P_{1}} \hat{P}_{r s_{1}}+\sum_{s_{2}=1_{2}}^{N E L P_{2}} \hat{P}_{r s_{2}}+\cdots+\sum_{s_{2}=1_{p}}^{N E L P_{p}} \hat{P}_{r s_{p}}, B_{s r}=\sum_{s_{1}=1_{1}}^{N E L P_{1}} \hat{P}_{s_{1} r}+\sum_{s_{2}=1_{2}}^{N E L P_{2}} \hat{P}_{s_{2} r}+\cdots+\sum_{s_{2}=1_{p}}^{N E L P_{p}} \hat{P}_{s_{p} r}, \\
D_{s}=\sum_{s_{1}=1_{1}}^{N E L P_{1}} P_{D s_{1}}+\sum_{s_{2}=1_{2}}^{N E L P_{2}} P_{D s_{2}}+\cdots+\sum_{s_{2}=1_{p}}^{N E L P_{p}} P_{D s_{p}} .
\end{gathered}
$$

Note that if the smart plate system is under input dynamic force on particular node, the parameters $v(j \omega) /-\omega^{2} w_{\text {base }} e^{j \omega t}$ and $\hat{Q}_{r}$ can be replaced with $v(j \omega) / F_{c}(j \omega)$ and $-\varphi_{r}$, respectively. The next equations will also use the same procedure.

The power harvesting multimode FRF related to the input transverse acceleration can be formulated as,

$$
\frac{P_{p}(j \omega)}{\left(-\omega^{2} w_{\text {base }} e^{j \omega t}\right)^{2}}=\left[\frac{\frac{1}{\sqrt{R_{\text {load }}}} \sum_{r=1}^{N D O F} \frac{j \omega B_{s r} \hat{Q}_{r}}{A_{r}}}{j \omega D_{s}-C-\sum_{r=1}^{N D O F} \frac{j \omega B_{r s} B_{s r}}{A_{r}}}\right]^{2} .
$$

Moreover, the transverse displacement multimode FRF related to the input transverse acceleration can be obtained as,

$$
\frac{a_{r}(j \omega)}{-\omega^{2} w_{\text {base }} e^{j \omega t}}=-\frac{1}{A_{r}}\left(\frac{\left(j \omega D_{s}-C\right) \hat{Q}_{r}}{j \omega D_{s}-C-\sum_{r=1}^{N D O F} \frac{j \omega B_{r s} B_{s r}}{A_{r}}}\right) .
$$

In terms of Eqs. (7) and (21), the characteristic transverse motion of the structure can be reformulated to give,

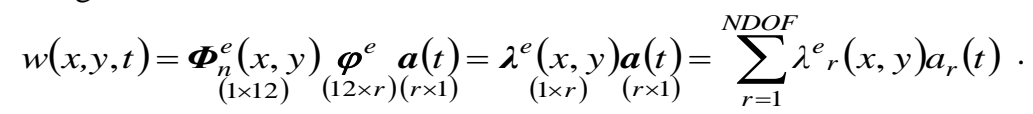

The relative transverse displacement related to the input base acceleration at any position along the smart plate structure $(x, y)$ can be formulated using Eqs. (37) and (38) as,

$$
\frac{w(x, y, j \omega)}{-\omega^{2} w_{\text {base }} e^{j \omega t}}=-\sum_{r=1}^{N D O F}\left[\frac{\frac{\left(j \omega D_{s}-C\right) \hat{Q}_{r} \lambda^{e} r(x, y)}{A_{r}}}{j \omega D_{s}-C-\sum_{r=1}^{N D O F} \frac{j \omega B_{r s} B_{s r}}{A_{r}}}\right] .
$$


The absolute transverse displacement and velocity FRFs can also be formulated using similar equations given in [31].

\subsection{The solution form of series piezoelectric/electrode segment connections}

By considering the segmented piezoelectric/electrode patterns with the electrical series connection as shown in Fig. 2b, the multi-mode FRFs show different equation as given in the parallel connection. Here, Eqs (24a)-(24c) and (29a)-(29c) associated with Eqs. (31a)-(31b) and (34) can be solved simultaneously using Laplace transform and the results of which can be further manipulated and simplified to give the electrical voltage multimode frequency response functions as,

$$
\frac{v(j \omega)}{-\omega^{2} w_{\text {base }} e^{j \omega \mathrm{t}}}=\frac{\sum_{r=1}^{N D O F} \frac{\hat{B}_{s r} \hat{Q}_{r}}{A_{r}-E_{s r}}}{-\frac{C \hat{D}_{s}}{j \omega}+1-\sum_{r=1}^{N D O F} \frac{\frac{\hat{B}_{r s} \hat{B}_{s r}}{\hat{D}_{s}}}{A_{r}-E_{s r}}},
$$

where

$$
\begin{aligned}
& \hat{B}_{r s}=\frac{\sum_{s_{1}=1_{1}}^{N E L P} \hat{P}_{r s_{1}}}{\sum_{s_{1}=1_{1}}^{N E L P} P_{D s_{1}}}+\frac{\sum_{s_{2}=1_{2}}^{N E L P} \hat{P}_{r s_{2}}}{\sum_{s_{2}=1_{2}}^{N E L P} P_{D s_{2}}}+\cdots+\frac{\sum_{s_{p}=1_{p}}^{N E L P} \hat{P}_{r s_{p}}}{\sum_{s_{p}=1_{p}}^{N E L P} P_{D s_{p}}}, \hat{B}_{s r}=\frac{\sum_{s_{1}=1_{1}}^{N E L P} \hat{P}_{s_{1} r}}{\sum_{s_{1}=1_{1}}^{N E L P} P_{D s_{1}}}+\frac{\sum_{s_{2}=1_{2}}^{N E L P} \hat{P}_{s_{2} r}}{\sum_{s_{2}=1_{2}}^{N E L P} P_{D s_{2}}}+\cdots+\frac{\sum_{s_{p}=1_{p}}^{N E L P} \hat{P}_{s_{p} r} r}{\sum_{s_{p}=1_{p}}^{N E L P} P_{D s_{p}}}, \\
& \hat{D}_{s}=\frac{1}{\sum^{N E L P} P_{D}}+\frac{1}{\sum_{s_{1}}^{N E L P} P_{D s_{2}}}+\cdots+\frac{1}{\sum^{N E L P_{p}} P_{D_{p}}}, \\
& \sum_{s_{1}=1_{1}} P_{D s_{1}} \quad \sum_{s_{2}=1_{2}}^{2} P_{D s_{2}} \quad \sum_{s_{p}=1_{p}}^{p} P_{D s_{p}}
\end{aligned}
$$

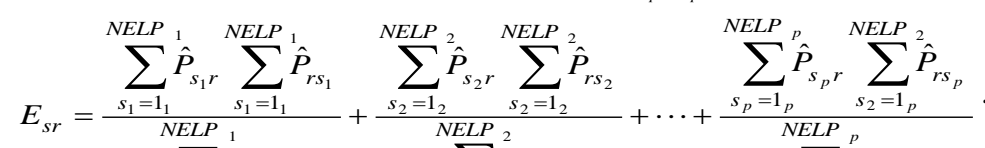

$$
\begin{aligned}
& \sum_{s_{1}=1_{1}}^{N E L P} P_{D s_{1}} \quad \sum_{s_{2}=1_{2}}^{2} P_{D s_{2}} \quad \sum_{s_{p}=1_{p}}^{p} P_{D s_{p}}
\end{aligned}
$$

The power harvesting multimode FRF can also be formulated to give,

$$
\frac{P_{p}(j \omega)}{\left(-\omega^{2} w_{\text {base }} e^{j \omega t}\right)^{2}}=\left[\frac{1}{\sqrt{R_{\text {load }}}} \frac{v(j \omega)}{-\omega^{2} w_{\text {base }} e^{j \omega t}}\right]^{2} .
$$

The multimode relative transverse displacement at any position along the smart plate structure $(x, y)$ can be formulated to give,

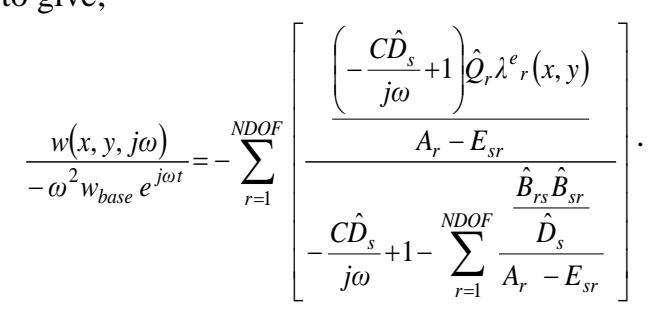

As shown in Eq. (42), the absolute transverse displacement and velocity FRFs can be formulated using similar equations in [31].

4.5 The solution form of mixed series-parallel piezoelectric/electrode segment connections

As shown in Fig. 2c, the multimode FRFs can be formulated using the combination between series and parallel piezoelectric/electrode segment connections. After manipulation and simplification, the voltage multimode FRFs can be formulated using Eqs. (24a)-(24c) and (29a)-(29c) associated with Eqs. (32a)-(32c), (33a)-(33b) and (34) to give, 


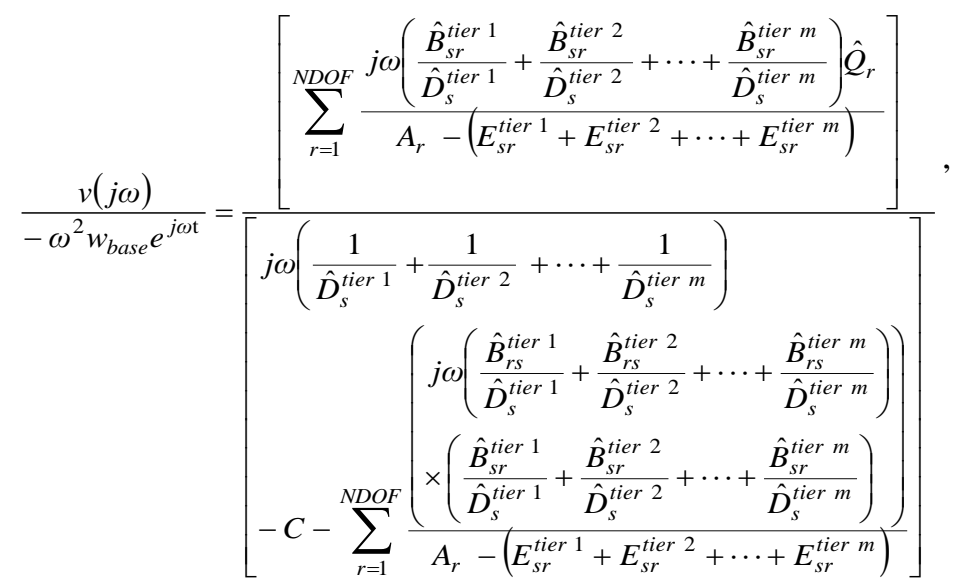

where

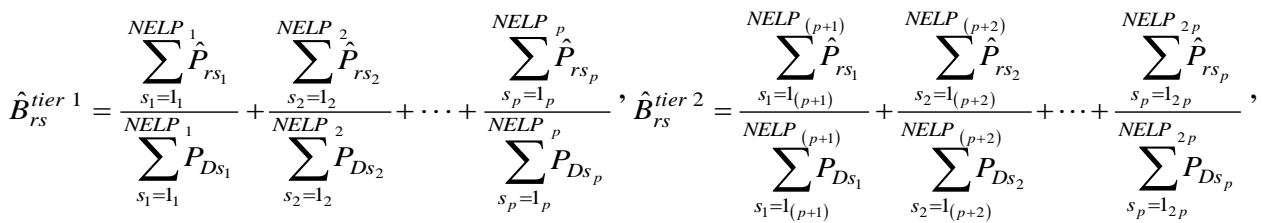

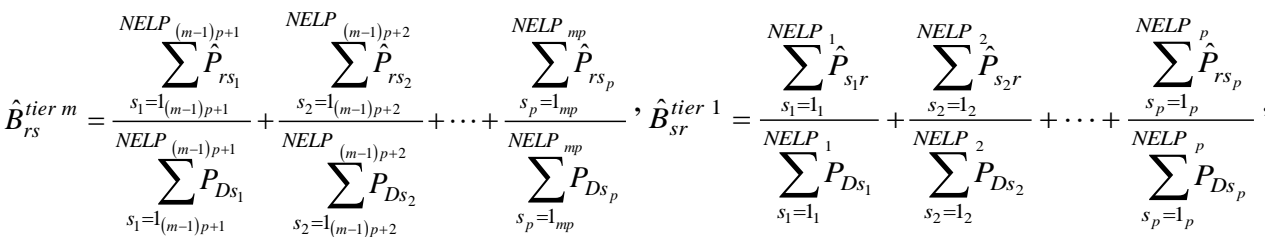

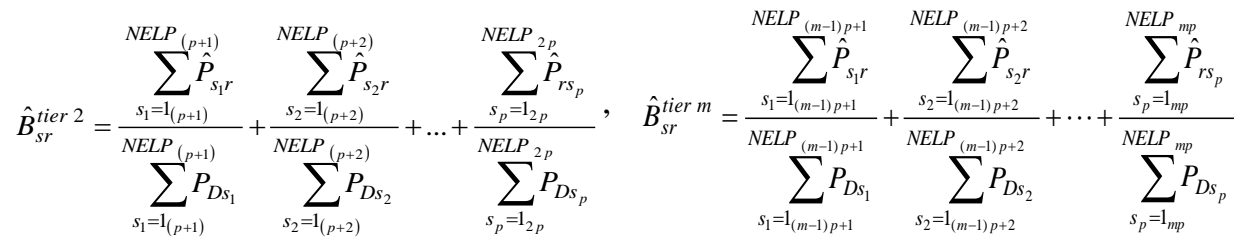

$$
\begin{aligned}
& \hat{D}_{s}^{\text {tier } 1}=\frac{1}{\sum_{s_{1}=1_{1}}^{N E L P} P_{D s_{1}}}+\frac{1}{\sum_{s_{2}=1_{2}}^{N E L P_{2}} P_{D s_{2}}}+\cdots+\frac{1}{\sum_{s_{p}=1_{p}}^{N E L P} P_{D s_{p}}^{p}}, \hat{D}_{s}^{\text {tier } 2}=\frac{1}{\sum_{s_{1}=1_{(p+1)}}^{N E L P} P_{D s_{1}}}+\frac{1}{\sum_{s_{2}=2_{(p+2)}}^{N E L P} P_{D s_{2}}}+\cdots+\frac{1}{\sum_{s_{p}=1_{2 p}}^{N E L P} P_{D s_{p}}}, \\
& \hat{D}_{s}^{\text {tier } m}=\frac{1}{\sum_{\left.s_{1}=1_{(m-1)}\right)}^{N E+1}}+\frac{1}{\sum_{D s_{1}}}+\frac{1}{\sum_{\left.s_{2}=1_{(m-1)}\right)+2}^{N E P^{\prime}} P_{D s_{2}}}+\cdots+\frac{1}{\sum_{s_{p}=1_{m p}}^{N E L P} P_{D s_{p}}}, \\
& E_{s r}^{\text {tier } 1}=\frac{\sum_{s_{1}=1_{1}}^{N E L P} \hat{P}_{s_{1} r} \sum_{s_{1}=1_{1}}^{N E L P} \hat{P}_{r s_{1}}}{\sum_{s_{1}=1_{1}}^{N E L P} P_{D s_{1}}}+\frac{\sum_{s_{2}=1_{2}}^{N E L P} \hat{P}_{s_{2} r} \sum_{s_{2}=1_{2}}^{N E L P} \hat{P}_{r s_{2}}}{\sum_{s_{2}=1_{2}}^{N E L P} P_{D s_{2}}}+\cdots+\frac{\sum_{s_{p}=1_{p}}^{N E L P} \hat{P}_{s_{p} r} \sum_{s_{p}=1_{p}}^{N E L P} \hat{P}_{r s_{p}}}{\sum_{s_{p}=1_{p}}^{N E L P} P_{D s_{p}}},
\end{aligned}
$$

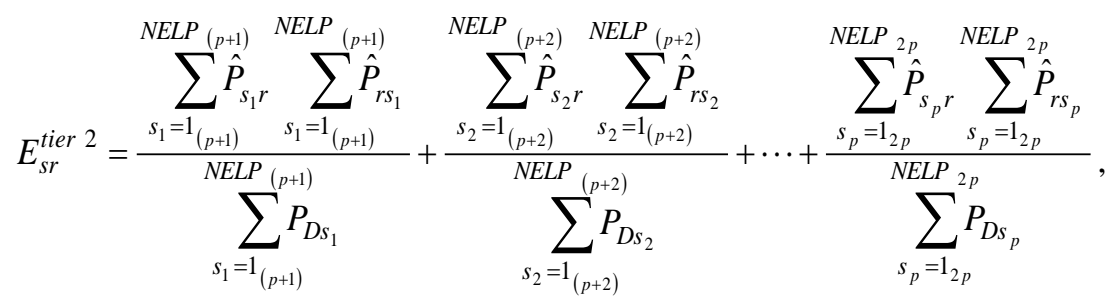




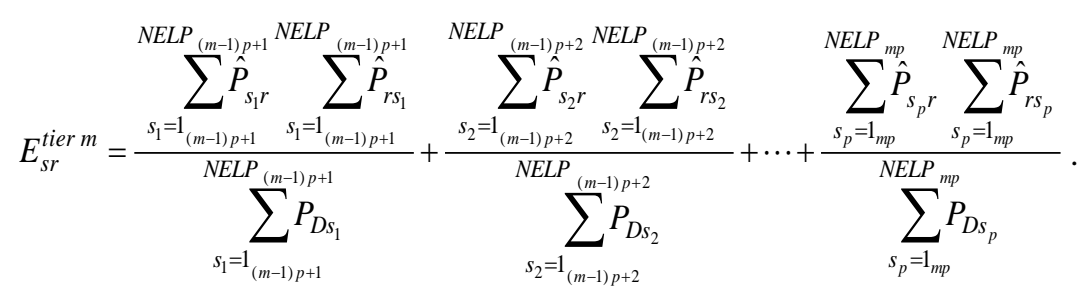

The power harvesting multimode FRF for multi-tier system can also be formulated using similar form of Eq. (41). Moreover, the relative transverse displacement multimode FRF can be formulated to give,

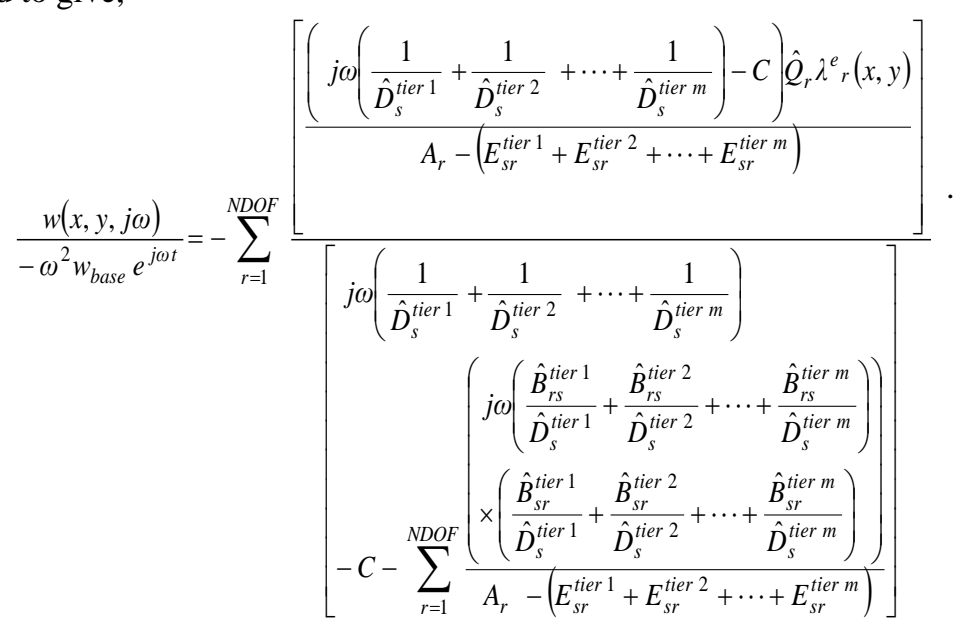

In terms of Eq. (44), the absolute transverse displacement and velocity FRFs can be formulated using similar equations in [31].

4.6 AC-DC harvesting circuit interface with segmented piezoelectric circuit connection patterns

Fig. 3 represents the time-waveform signal processes of the AC electrical voltage and the AC-DC full bridge rectifier with smoothing RC circuit from the piezoelectric element [33], [65]. For every positive half-cycle, each time the capacitor conducts for charging, the DC current can be generated due to the forward biased diodes D1 and D2. During the time for discharging due to the reverse biased diodes D3 and D4 , the capacitor voltage decays resulting in no current. For negative halfcycle, the diodes D1 and D2 becomes reverse biased and no conduct where diodes D3 and D4 turn forward biased and conduct. This process repeats every half-cycle.

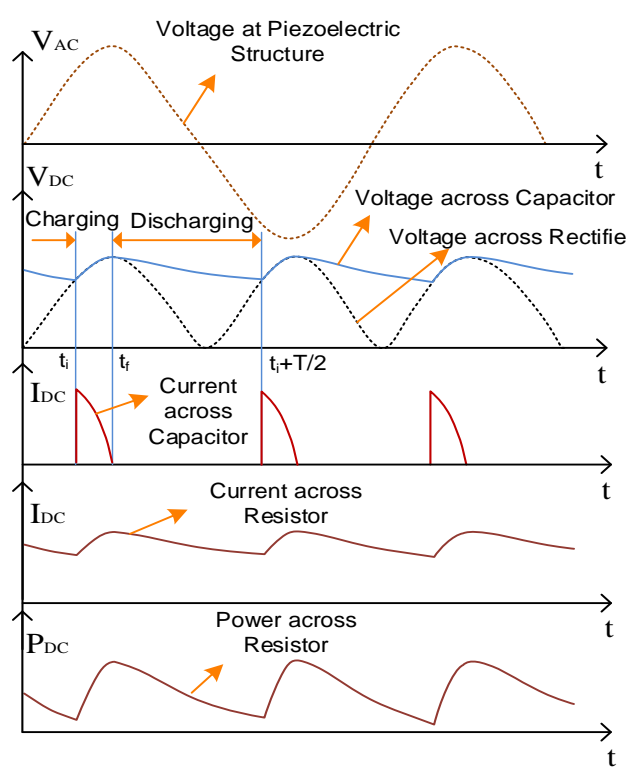

Fig. 3 Time waveforms of standard harvesting circuit 
The waveforms as shown in Fig. 3 obviously represent the process of the signal outputs through the harvesting circuit from the piezoelectric component due to the dynamic motion. If there is no motion to the piezoelectric system, the waveforms may not show DC signals. That means that the waveforms (DC signal) obviously depend on the signal from the piezoelectric itself as the main source of the electrical energy (AC signal).

4.6.1 Current flowing with interval $t_{i}<t<t_{f}$ indicating the charging time every half-cycle of the signal waveform.

In this section, the option of each electrical connection pattern (parallel, series, and mixed seriesparallel piezoelectric/electrode segment connections) can be performed using the computational charging process via the harvesting circuit interface. The signal outputs as shown in Fig. 3 have different time-waveform responses depending upon each connection pattern of the piezoelectric segments.

a. DC signal outputs for the parallel piezoelectriclelectrode segment connection During period of charging, the DC signal time response can be formulated using Eqs. (24a)-(24c) and (29a)-(29c) associated with Eqs. (30a)-(30b) and (34). The simultaneous results can be formulated to give the following state space representation for the multi-mode response system as,

$$
\frac{\mathrm{d}}{\mathrm{d} t}\left\{\begin{array}{c}
a_{1} \\
a_{2} \\
\cdot \\
\cdot \\
a_{r} \\
\dot{a}_{1} \\
\dot{a}_{2} \\
\cdot \\
\cdot \\
\dot{a}_{r} \\
v_{d}
\end{array}\right\}=\left[\begin{array}{c}
\dot{w}_{1} \\
\dot{w}_{2} \\
\cdot \\
\cdot \\
\dot{w}_{r} \\
-2 \zeta_{1} \omega_{1} \dot{a}_{1}(t)-\omega_{1}^{2} a_{1}(t)-B_{1 s} v_{d}-\hat{Q}_{1} \ddot{w}_{\text {base }}(t) \\
-2 \zeta_{2} \omega_{2} \dot{a}_{2}(t)-\omega_{2}^{2} a_{2}(t)-B_{2 s} v_{d}-\hat{Q}_{2} \ddot{w}_{\text {base }}(t) \\
\cdot \\
\cdot \zeta_{r} \omega_{r} \dot{a}_{r}(t)-\omega_{r}{ }^{2} a_{r}(t)-B_{r s} v_{d}-\hat{Q}_{r} \ddot{w}_{\text {base }}(t) \\
-\sum_{r=1}^{N D O F} B_{s r} \dot{a}_{r}(t)+\frac{v_{d}}{R_{d}} \\
D_{s}-C_{d}
\end{array}\right] .
$$

-It should be noted that parameters $B_{r s}, B_{s r}$ and $D_{s}$ can be found in Eqs. (35c)-(35d).

\section{b. DC signal outputs for the series piezoelectric/electrode segment connections}

Corresponding with Eqs (24a)-(24c) and (29a)-(29c) associated with Eqs. (31a)-(31b) and (34), the state space representation for the multi-mode response system for the period of charging can be formulated after manipulation and simplification,

$$
\frac{\mathrm{d}}{\mathrm{d} t}\left\{\begin{array}{c}
a_{1} \\
a_{2} \\
\cdot \\
a_{r} \\
\dot{a}_{1} \\
\dot{a}_{2} \\
\cdot \\
\cdot \\
\dot{a}_{r} \\
v_{d}
\end{array}\right\}=\left[\begin{array}{c}
\dot{w}_{1} \\
\dot{w}_{2} \\
\cdot \\
\cdot \\
\dot{w}_{r} \\
-2 \zeta_{1} \omega_{1} \dot{a}_{1}(t)-\omega_{1}^{2} a_{1}(t)+E_{s 1} a_{1}(t)-\frac{\hat{B}_{s 1} v_{d}}{\hat{D}_{s}}-\hat{Q}_{1} \ddot{w}_{\text {base }}(t) \\
-2 \zeta_{2} \omega_{2} \dot{a}_{2}(t)-\omega_{2}{ }^{2} a_{2}(t)+E_{s 2} a_{2}(t)-\frac{\hat{B}_{s 2} v_{d}}{\hat{D}_{s}}-\hat{Q}_{2} \ddot{w}_{\text {base }}(t) \\
\cdot \\
\cdot \\
\sum_{r=1}^{N D O F} \hat{B}_{r s} \dot{a}_{r}(t)-\left(\frac{\hat{D}_{s}}{R_{d}}-1\right) v_{d} \\
\hat{D}_{s} C_{d}
\end{array}\right]
$$


Note that parameters $\hat{B}_{r s}, \hat{B}_{s r}, \hat{D}_{s}$ and $E_{s r}$ can be found in Eqs. (40b)-(40d).

\section{c. DC signal outputs for the mixed series-parallel piezoelectric/electrode segment connections}

After manipulation and simplification, the state space representation for the multi-mode response system for the period of charging can be formulated using Eqs. (24a)-(24c) and (29a)-(29c) associated with Eqs. (32a)-(32c), (33a)-(33b) and (34) to give,

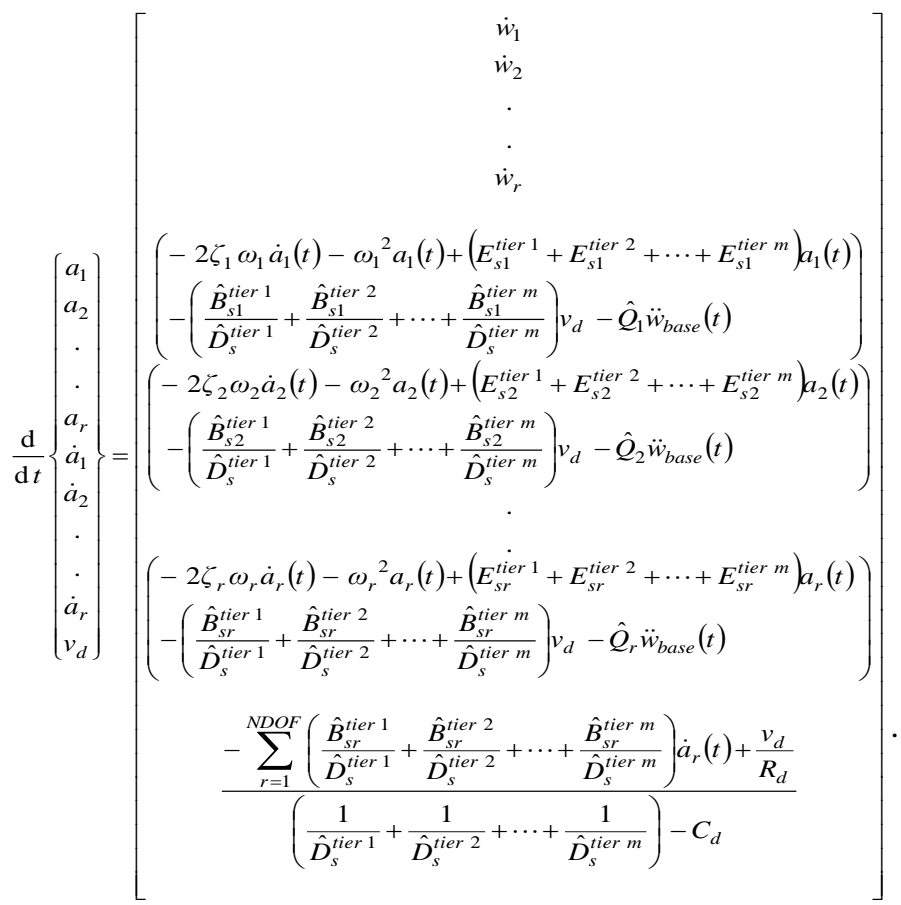

Note that all parameters with the superscript tier can be found in Eqs. (43b)-(43i).

4.6.2 Current flowing with interval $t_{f}<t<t_{i}+T / 2$ indicating the discharging times every halfcycle of the signal waveform.

Since each connection pattern of the piezoelectric/electrode segments has the same harvesting circuit for discharging process, the harvesting circuit from Eq. (34) can be reduced as,

$$
C_{d} \dot{v}_{d}+\frac{v_{d}}{R_{d}}=0
$$

The solution form of (48) can be stated as,

$$
v_{d}(t)=v_{d}\left(t_{f}\right) \exp \left(\frac{-\left(t-t_{f}\right)}{C_{d} R_{d}}\right) .
$$

It should be noted that during charging and discharging processes, the current and voltage time signal waveform outputs for the parallel, series, and mixed series-parallel piezoelectric/electrode segment connections can be simulated using Eqs. (45) and (49), Eqs. (46) and (49), and Eqs. (47) and (49), respectively. Once collecting the signal data from some parameters during charging and discharging processes using these equations, the DC currents across the resistor and capacitor including DC power output across the resistor as shown in Fig. 3 can be formulated respectively as,

$$
I_{D C_{-} R_{d}}=\frac{v_{d}}{R_{d}}, I_{D C_{-} C_{d}}=\dot{v}_{d} C_{d}, P_{p_{-} R_{d}}=v_{d} I_{D C_{-} R_{d}} .
$$

Note that the parameter $\dot{v}_{d}$ at DC signal output for the parallel segment connection can be formulated by taking the last part of Eq. (45), giving, 


$$
\dot{v}_{d}=\left|\frac{-\sum_{r=1}^{N D O F} B_{s r} \dot{a}_{r}(t)+\frac{v_{d}}{R_{d}}}{D_{s}-C_{d}}\right|=\left\{\begin{array}{l}
-\dot{v}_{d} \text { if } \dot{v}_{d}<0 \\
\dot{v}_{d} \text { if } \dot{v}_{d} \geq 0
\end{array} .\right.
$$

Also note that the modulus of $\dot{v}_{d}$ was utilized due to using AC-DC full bridge rectifier as shown in Fig. 3. For series segment connection, the parameter $\dot{v}_{d}$ at DC signal output can be formulated as,

$$
\dot{v}_{d}=\left|\frac{\sum_{r=1}^{N D O F} \hat{B}_{r s} \dot{a}_{r}(t)-\left(\frac{\hat{D}_{s}}{R_{d}}-1\right) v_{d}}{\hat{D}_{s} C_{d}}\right|=\left\{\begin{array}{l}
-\dot{v}_{d} \text { if } \dot{v}_{d}<0 \\
\dot{v}_{d} \text { if } \dot{v}_{d} \geq 0
\end{array} .\right.
$$

For mixed series-parallel connection, the parameter $\dot{v}_{d}$ at DC signal output becomes,

$$
\dot{v}_{d}=\left|\frac{-\sum_{r=1}^{N D O F}\left(\frac{\hat{B}_{s r}^{\text {tier } 1}}{\hat{D}_{s}^{\text {tier } 1}}+\frac{\hat{B}_{s r}^{\text {tier } 2}}{\hat{D}_{s}^{\text {tier } 2}}+\cdots+\frac{\hat{B}_{s r}^{\text {tier } m}}{\hat{D}_{s}^{\text {tier } m}}\right) \dot{a}_{r}(t)+\frac{v_{d}}{R_{d}}}{\left(\frac{1}{\hat{D}_{s}^{\text {tier } 1}}+\frac{1}{\hat{D}_{s}^{\text {tier } 2}}+\cdots+\frac{1}{\hat{D}_{s}^{\text {tier } m}}\right)-C_{d}}\right|=\left\{\begin{array}{l}
-\dot{v}_{d} \text { if } \dot{v}_{d}<0 \\
\dot{v}_{d} \text { if } \dot{v}_{d} \geq 0
\end{array} .\right.
$$

\section{Results and discussion}

This section discusses the effect of the multiple electrode segments onto piezoelectric plate structures under input base excitation using the three different segment connection patterns. Note that since the main contribution of this paper is in proposing a technique of the multi-segments with the circuit connection patterns, the rectangular finite element model has been utilized for simplicity of the geometrical analysis. For this reason, the typical thin cantilever plate structure under base excitation provides an effective design for generating high power output in the lower frequency range. For the future work using different shapes of the plate element and complex geometrical structure, the NURBS (Non-Uniform Rational B-Splines)-based isogeometric finite element analysis will be employed for investigating the optimal power output at particular segments of the system. Although the electromechanical dynamic equations can be used for the multiple piezoelectric segments, the multiple electrode segments have been chosen for analyzing the parametric numerical case studies due to giving the physical insights of the electromechanical system response behaviors and practical implementations. At this point, the numerical studies also discuss the effective and potential options from the three different circuit connection patterns connected from the variable segmented electrode system of the smart plate structures. This section also discusses the convergence criteria, mode shapes, and frequency analysis with the effect of strain nodes and input dynamic force followed by the DC time signal waveforms. The material properties of the smart plate structure that exhibits isotropic behavior are given in table 1 . The piezoelectric material used here was made from PZT-5A as given from efunda [66] where its properties were reduced from the plane-stress $3 \mathrm{D}$ to $2 \mathrm{D}$ components in order to obtain the correct data from the piezoelectric plate system. The geometrical smart plate structures under input excitation of $1 \mathrm{~ms}^{-2}$ were set to $150 \mathrm{~mm}$ length $(L)$ and $80 \mathrm{~mm}$ width (b) with the piezoelectric thickness $\left(h^{(1)}\right)$ of $0.127 \mathrm{~mm}$ and the substructure (brass) thickness $\left(h^{(2)}\right)$ of $1 \mathrm{~mm}$.

In Fig. 4, smart plate structure under base excitation has twelve electrode segments and each of them consists of six electromechanical discretized elements. The segmented system can be arranged electrically in parallel, series, and mixed series-parallel connections as shown in Figs. 5a$5 \mathrm{c}$, respectively. It should be noted here that each connection can be controlled using the on-off switching techniques. For example, if the systems with the parallel and mixed series-parallel segment connections are controlled by the tiers 1 and 2 (double tier), the switches $S_{1}$ and $S_{2}$ will turn on where the other switches $S_{3}$ and $S_{4}$ turn off. For quadruple tier (all tiers), all switches will be active and so on. For series connection, if the tiers 1 and 2 are used, only switch $S_{2}$ will turn on. If quadruple tier (all tiers) are used, only switch $\mathrm{S}_{4}$ will turn on. Also note that the parallel and series electrode segment connections as shown in Figs. 5a-5b utilize the tier system whereas section 4.2 shows those particular connections without tier system because this section explores 
the electromechanical behavior using only the same segment pattern of the structural system. Again, the constitutive frequency equations in Sec. 4.2 (Figs. 2a-2b without tier) are the same for the analysis on this section. The reason is that they represent the general equations which can be used to the models with arbitrary piezoelectric or electrode segments located at any locations of the system.

Table 1. Properties of the piezoelectric plate structure

\begin{tabular}{ll|lc}
\hline Material properties & Piezoelectric & Material properties & Brass \\
\hline Reduced elastic stiffness coefficients & $69.49,24.32$, & Young's modulus & \\
$\bar{c}_{11}, \bar{c}_{12}=\bar{c}_{21}, \bar{c}_{22}, \bar{c}_{66}(\mathrm{GPa})$ & $69.49,22.57$ & & 105 \\
Density $\rho\left(\mathrm{kg} / \mathrm{m}^{3}\right)$ & 7750 & Poisson's ratio $v$ & 0.3 \\
Reduced piezoelectric constant & & Density $\rho\left(\mathrm{kg} / \mathrm{m}^{3}\right)$ & 9000 \\
$\bar{e}_{31}=\bar{e}_{32}\left(\mathrm{C} / \mathrm{m}^{2}\right)$ & -16.0413 & & \\
Reduced Permittivity $\bar{\varepsilon}_{33}^{S}(\mathrm{nF} / \mathrm{m})$ & 9.5657 & & \\
\hline
\end{tabular}

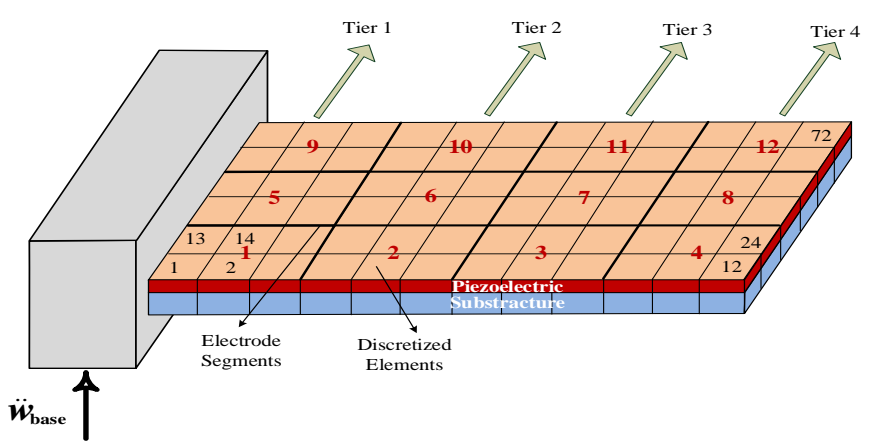

Fig. 4 The smart plate structure with the segmented electrodes and electromechanical discretization.

(a)

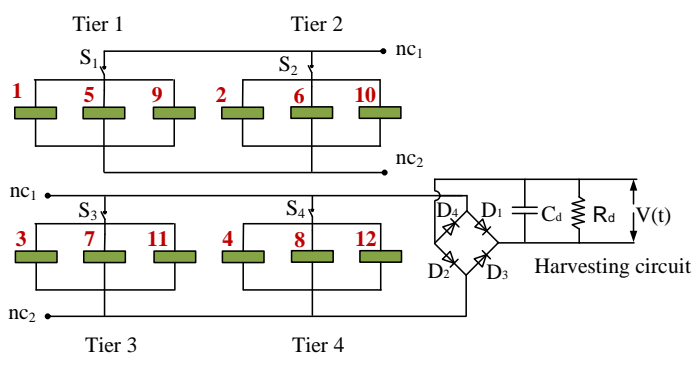

(c)

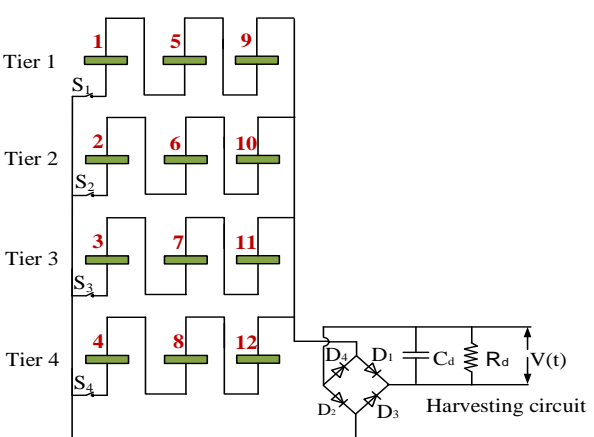

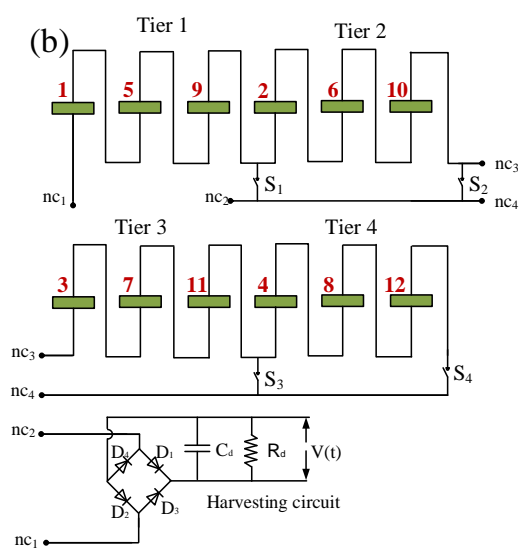

Fig. 5 The segment patterns with the tier system and the standard AC-DC circuit interfaces corresponding with the onoff switching technique: (a) the parallel electrode segment connections, (b) the series electrode segment connection, and (c) the mixed series-parallel electrode segment. 
The convergence criteria for the first four modes obtained from the proposed numerical studies can be seen in Figs. 6a-6c. Increasing number of electromechanical elements in $x$ - and $y$-axes for each mode can give the variable resonance frequency approaching the equal value. Since the convergence criteria is one of the essential aspects for studying the accuracy of the numerical methods, the study shown here is that the higher modes normally require a higher number of elements. However, if lower modes $\left(1^{\text {st }}\right.$ and $2^{\text {nd }}$ modes $)$ are major concerned, a lower number of elements with the reasonable and careful choice will be sufficient. This can be seen in Figs. 6a and $6 \mathrm{~b}$ where the predominant resonance frequencies tend to give the same values. At this case, the 72 electromechanical discretized elements with the total of 273-degree-of-freedom for the first four modes have shown sufficiently accuracy for further analyzing the frequency responses and time signal waveforms. As shown in Figs.7a-7d, the first four mode shapes using the discretized element number can also be identified. It is clearly seen that the first and third modes represent the first and second transverse modes, respectively. However, the second and fourth modes show the first and second twisting modes, respectively.
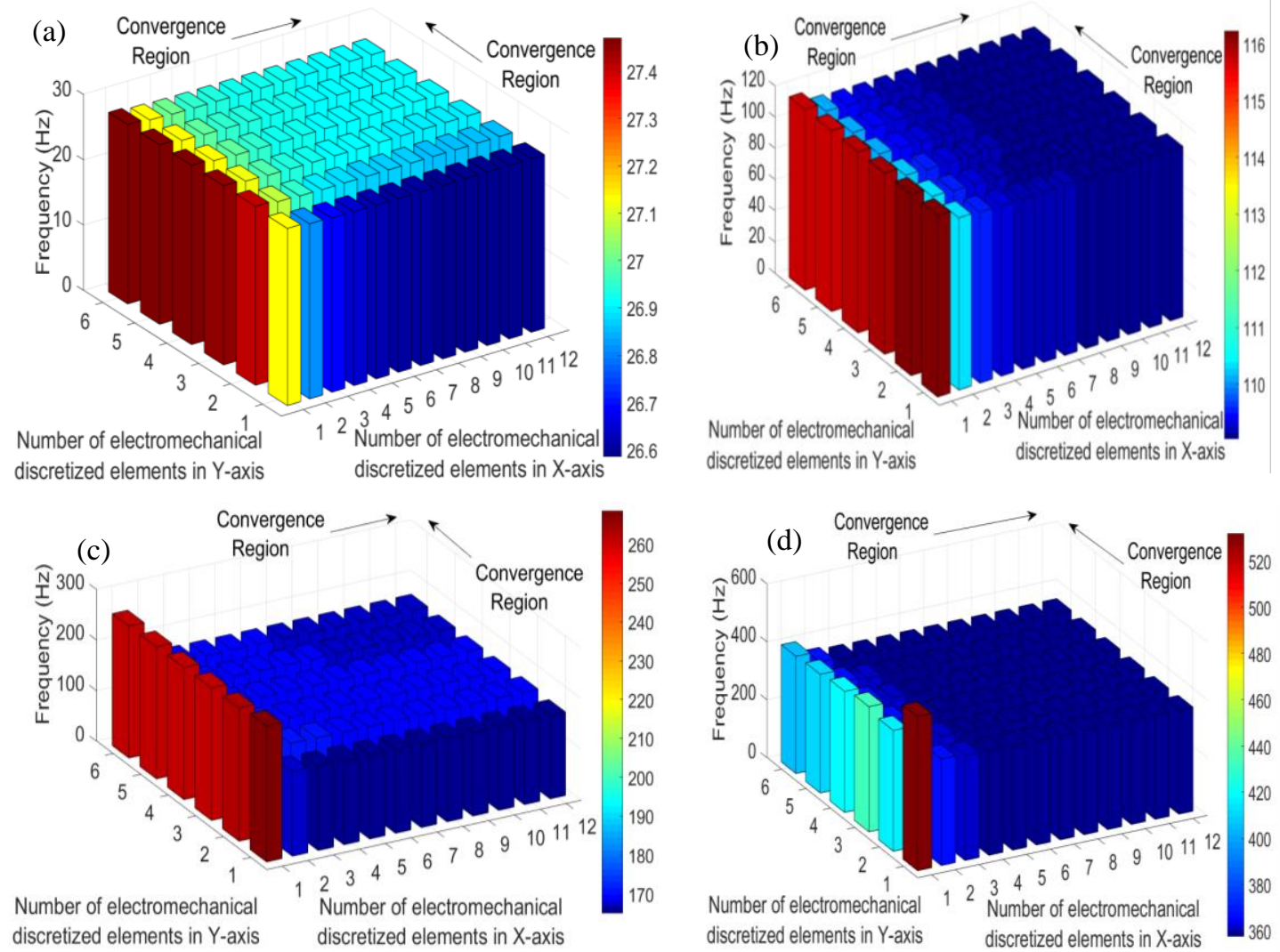

Fig. 6 Convergence studies based on the discretized elements: (a) the first resonance frequency, (b) the second resonance frequency, (c) the third resonance frequency and (d) the fourth resonance frequency.

(a)

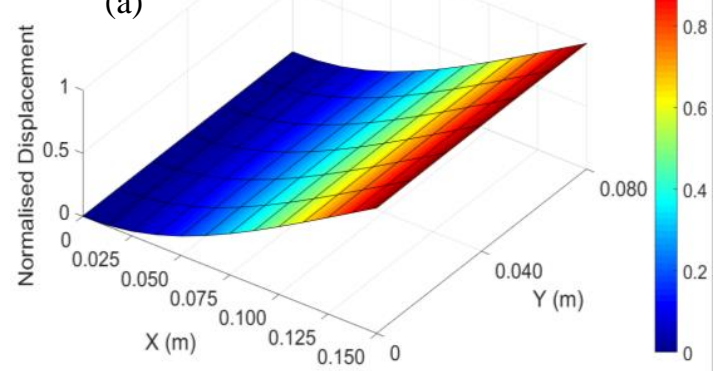

(b)

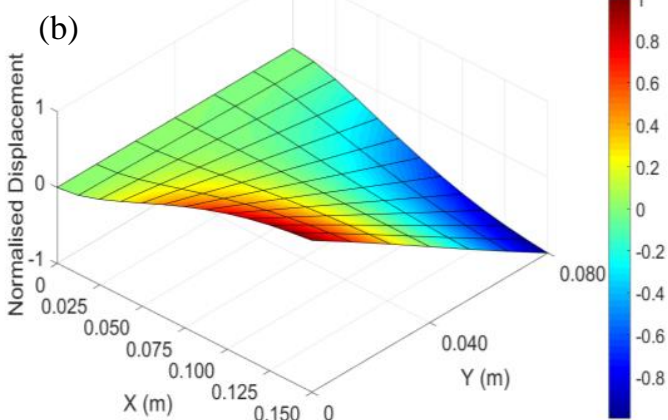


(c)

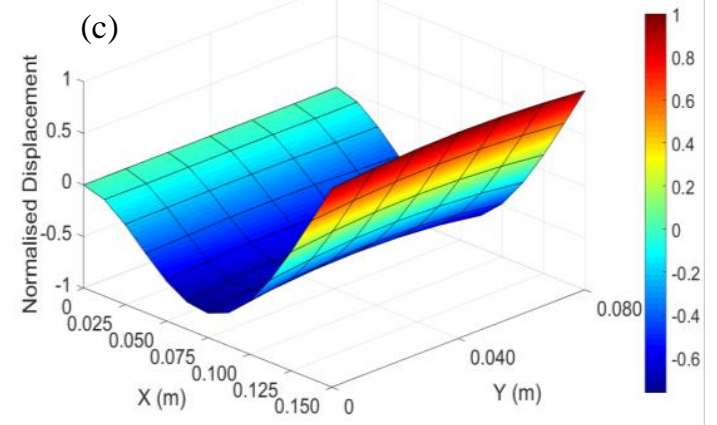

(d)

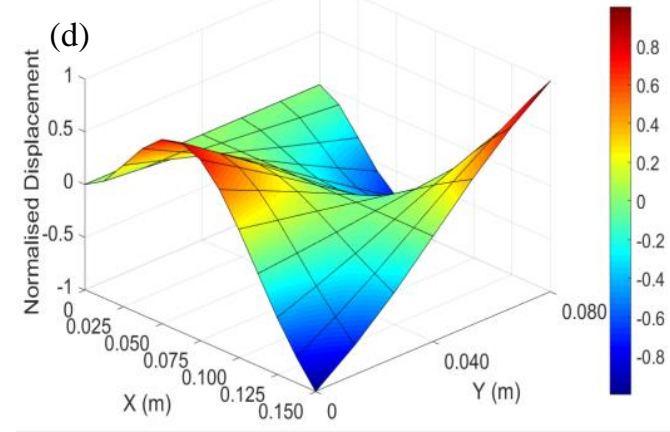

Fig. 7 Normalized displacement: (a) the first mode shape $(26.914 \mathrm{~Hz})$, (b) the second mode shape $(109.080 \mathrm{~Hz})$, (c) the third mode shape $(167.918 \mathrm{~Hz})$ and $(\mathrm{d})$ the fourth mode shape $(358.115 \mathrm{~Hz})$.

With the distributed electrode covered on the entire piezoelectric structure, the power output frequency response behavior under variable load resistance as shown in Fig. 8c only represents the two peaks of resonances indicating the first and second transverse modes. As expected, the first and second twisting modes clearly disappear due to the strain nodes of those modes using the inflection point of the second order differential shape function of the strain-displacement relationship. It is important to note that if the parallel electrode segment system with active all tiers is implemented, the FRFs will give the same results as the system response with the distributed electrode segment. Also note that strain node regions as shown in Figs. 8a and 8b are located along the $x$-axis and both $x$ - and $y$-axes of the smart plate structure, respectively. The major reduction of the power outputs of the twisting modes are resulted from the tendency of equal strain distribution changes between the element regions with the blue and red colors creating asymmetry shape. As a result, the electric current for the entire elements reduces significantly giving the effect of reduction of the power output. Indeed, the system with the input base excitation can predominantly create the transverse modes. As a result, the twisting modes even further disappear in the power output FRFs. However, further analysis using input dynamic force on certain node using certain electrode segment locations can give different perspective as discussed in the next stage.
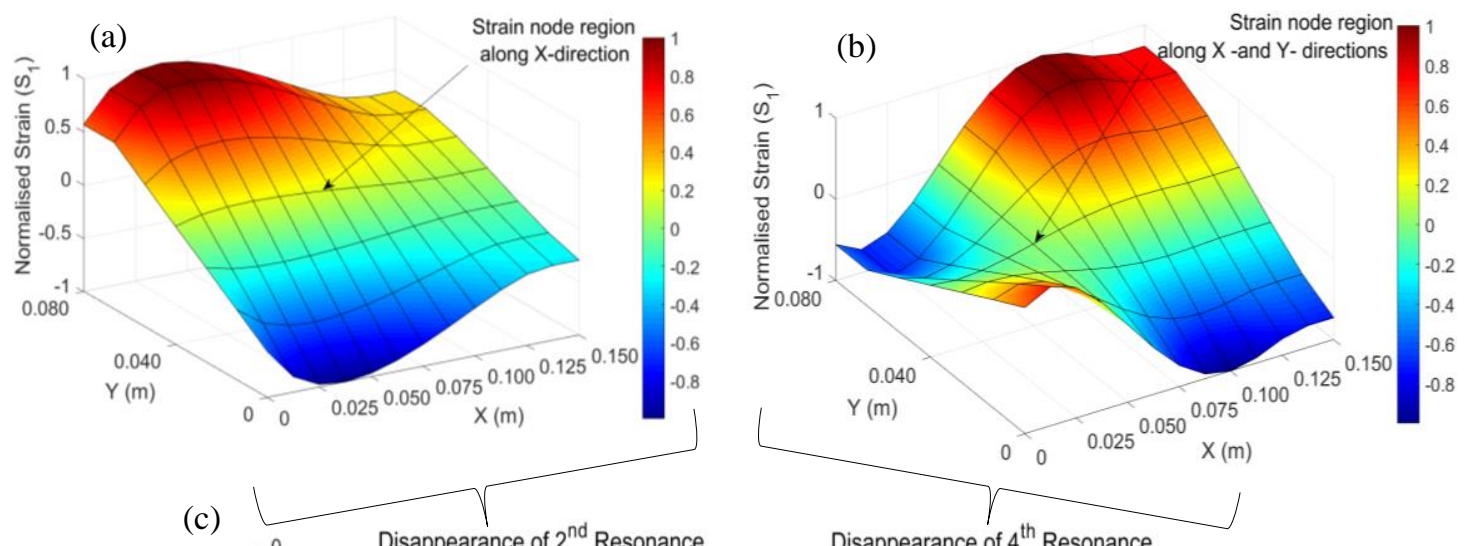

(c)
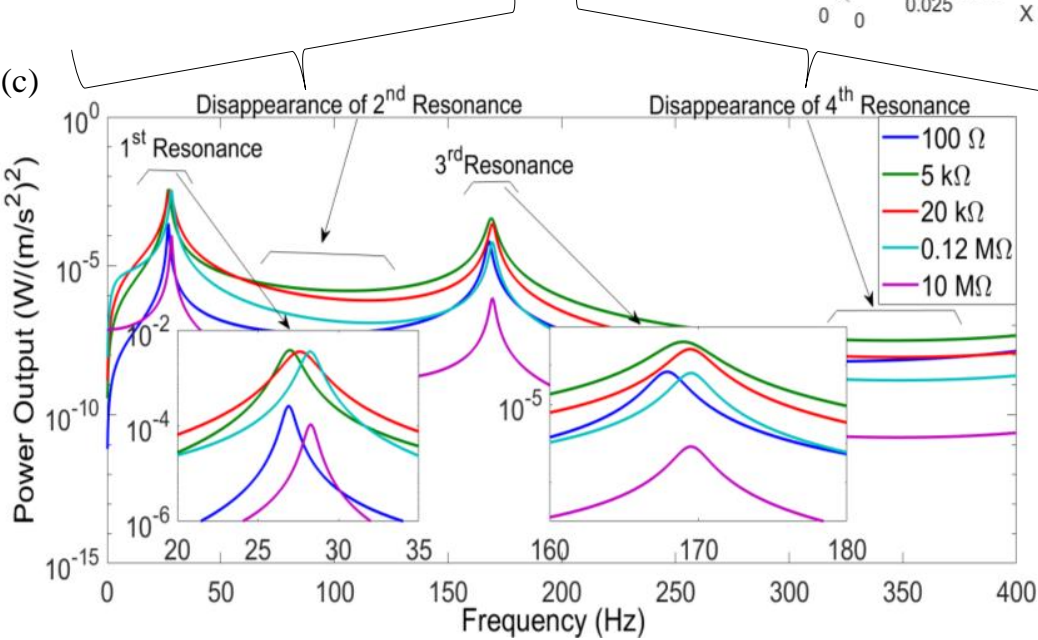

Fig. 8 the system responses : (a) the second strain mode shape (first twisting mode), (b) the fourth strain mode shape (second twisting mode) and (c) power harvesting FRFs with variable load resistance using the distributed electrode segment connection. 
With variation of the load resistance, the power FRFs of the first mode shift from short circuit to open circuit conditions. The lowest amplitudes are normally found from those conditions. However, higher amplitudes can be achieved gradually as being away from those conditions. Similarly, the power output at the third mode also varies from short circuit to open circuit conditions, just being a slight different trend to that of the first mode.

Figs. 9a-9b show variation of the power outputs from series and parallel electrode segment connections. As mentioned previously, each tier consists of the electrode segments onto the piezoelectric structure that can be arranged electrically in series and parallel connections. The on-off switching techniques are used to control the implementation of each tier. Here, as shown in Figs. 9a$9 \mathrm{~b}$, the power outputs with the short and open circuit load resistances for both connection patterns tend to shift. Note that it is only a given example of using the short and open circuit load resistances since it can give a technical insight of shifting resonance frequency. Again, the first and second twisting modes clearly disappear for this case. Instantly view to the figure, the trends for both connection patterns seem to be similar where each tier looks different. However, with further investigation to each tier, the power outputs for the parallel electrode segments as shown in Fig. 9d increase with increasing the tier numbers and the same resonance frequency. That situation also occurs for the third mode (second transverse mode) except double tier. In the opposite behavior, the power outputs using series electrode segments for both modes as shown in Fig. 9e clearly show the reduction of amplitudes while increasing the tier numbers. The inherent system of the material involves piezoelectric couplings and internal capacitance contributing the electromechanical damping and electromechanical stiffness [31].
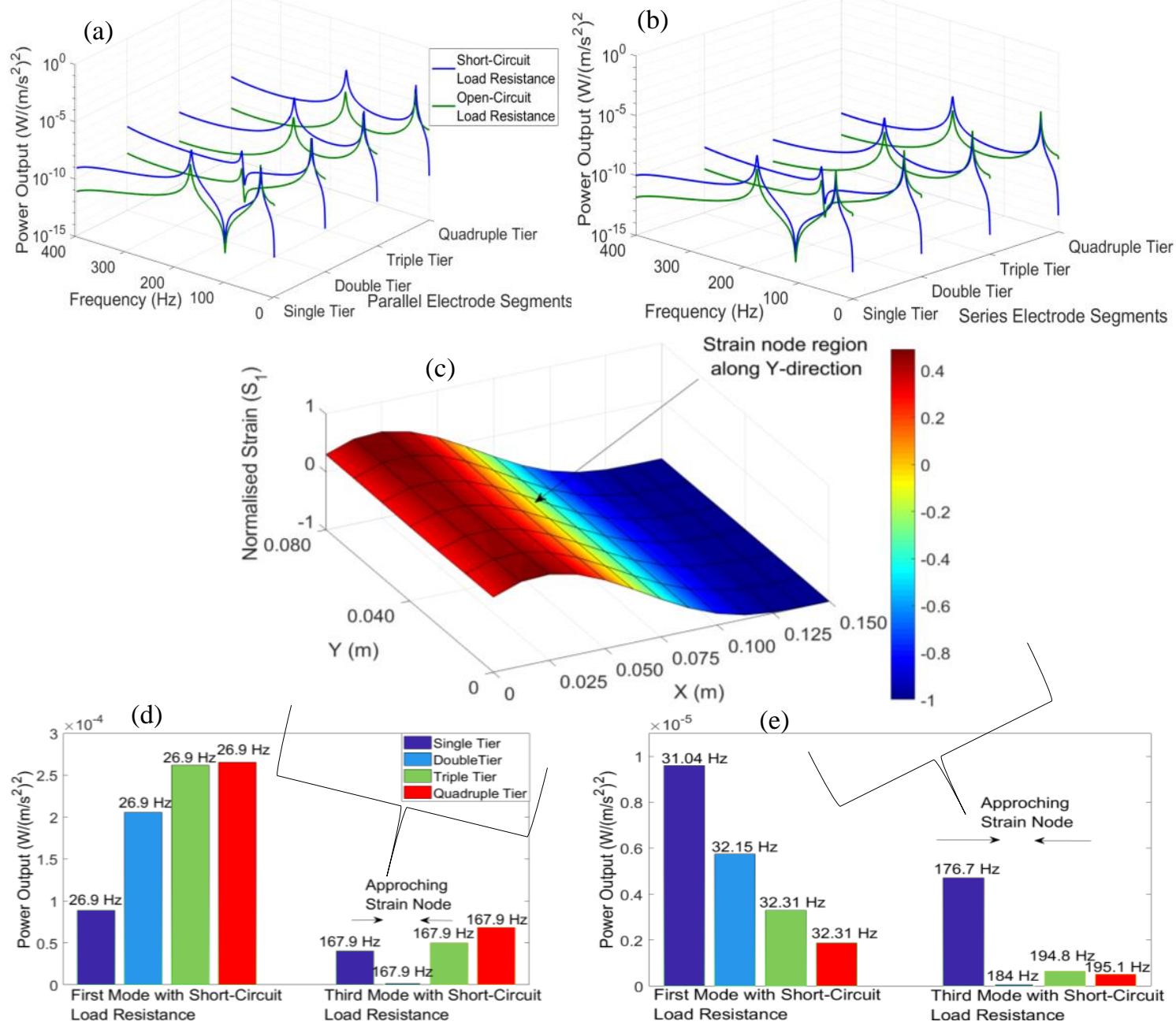

Fig 9 the system responses using various tier networks: (a) power harvesting FRFs under short and open circuit load resistances using the parallel electrode segment connections, (b) power harvesting FRFs under short and open circuit load resistances using the series electrode segment connection, (c) the third strain mode shape, (d) power amplitude and frequency comparisons with the parallel electrode segment connections, (e) power amplitude and frequency comparisons using the series electrode segment connections. 

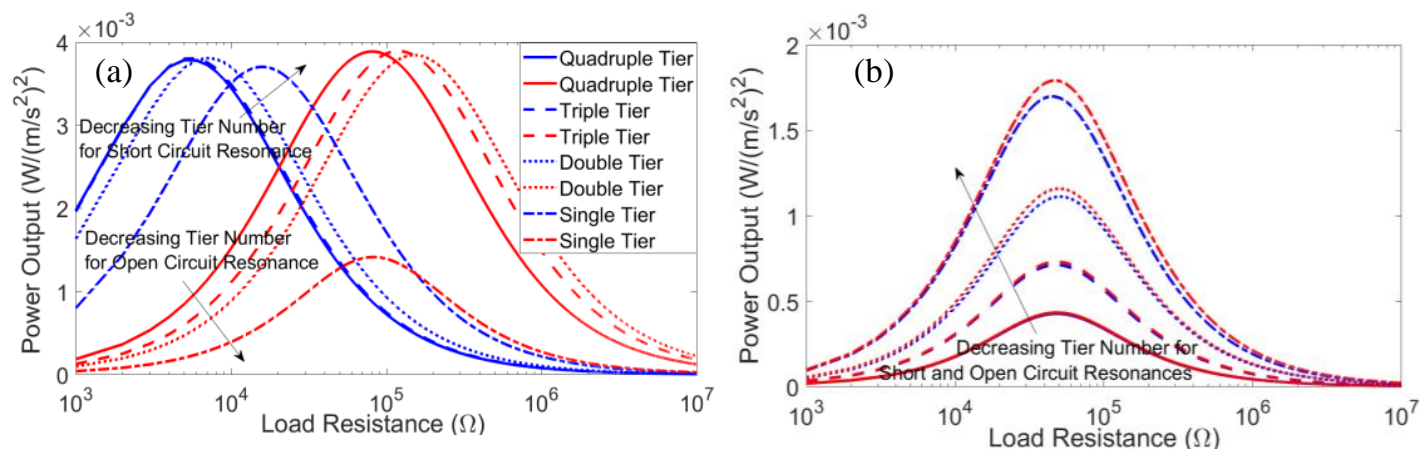

Fig. 10 Power outputs under the excitations of the short circuit and open circuit resonance frequencies using various tier networks: (a) the parallel electrode segment connections and (b) the series electrode segment connection.

However, it is further proved here that the physical behavior of changes of the electrical connection to the electrode segments also affects the electromechanical system of the smart plate structure. Slightly different results for the third mode can be seen in the double tier for both connection patterns where the power amplitudes reduce significantly due to the third strain mode (second transverse strain mode in Fig. 9c). This occurs because the portions of electrode segments for double tier strain node regions are located between 0 and $0.075 \mathrm{~m}$ in $\mathrm{x}$-axis. Note that the valley of the strain mode shows higher portion compared with the hill region. As a result, the portions of electrode segments for triple and quadruple tiers for parallel electrode segments show higher power amplitude regions. In contrast, lower power amplitude regions for series electrode segments occur at higher tiers. For that reason, next stage discusses the mixed series-parallel connections for optimizing the power output resonance and amplitude.

In different fashion as shown in Figs. 10a for the parallel electrode segments with various tier connections, the particular power outputs under the excitations of the short circuit and open circuit resonance frequencies show two optimum amplitudes. In general form, the power outputs under the short circuit and open circuit resonance excitations can give the same amplitude due to the coincidence of the particular load resistance which is so-called transitional or critical point. For example, the power outputs using triple tier as shown in the red and blue dash-lines will meet each other at the particular load resistance value. It is obvious to see that the power outputs with decreasing tier numbers under short circuit condition appear to shift their amplitudes using higher load resistance. Similarly, the power outputs with decreasing tier number under open circuit condition also shift except single tier as being the lowest value. The contribution of parallel electrode segment connection with quadruple or triple tier clearly gives higher power amplitude as shown in the excitations for both short and open circuit conditions. Unlike common responses given in Fig. 10a, the responses as shown in Fig. 10b for the series electrode segment connection with the various tier connections show differently. The power outputs under both conditions seem to be very close to each other and increase significantly with decreasing the tier numbers.

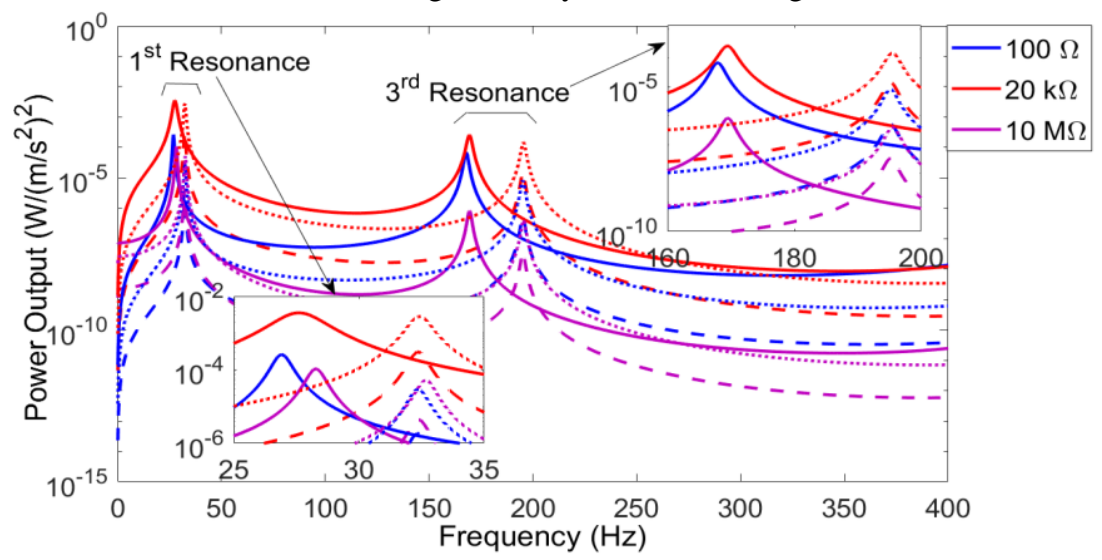

Fig. 11 Power harvesting FRFs using the quadruple tier network with the parallel electrode segment connections (solid lines), series electrode segment connections (dash lines) and mixed series-parallel electrode segment connection (dot lines).

This also shows a similar trend as given in Fig. 9e. However, in comparison with Fig. 10b, it is clearly seen that the power outputs using the parallel electrode segments seem to give a slight higher amplitude compared to that of response given from the series electrode segments. In Fig. 
11, the parallel, series and mixed series-parallel electrode segment connections with the chosen load resistances have been used for comparison using the quadruple tier. As shown, the power outputs generated from the parallel and mixed series-parallel electrode segment systems can give higher optimal amplitudes compared with the series electrode segment. However, when the resonance frequency of the smart plate structure is away from the ambient frequency, matching the two different frequency environments are essentially required so as to maintaining the optimal power output. For that reason, the shift of the power amplitude at particular frequency of the system can be potentially required using the mixed series-parallel electrode segment connection as being an effective option for this case. The shift of the power output frequency at the third mode appears to be more pronounced widening up to $25 \mathrm{~Hz}$.

In previous case studies, the systems under input base excitation have been used for analysis using the three different electrode segment connection patterns. It is clearly seen from the previous results that the second and fourth modes (first and second twisting modes) of the power outputs disappeared. The reason of such physical behavior has also been explained. However, this stage addresses of how to optimize those modes using the input dynamic force of $0.2 \mathrm{~N}$ at particular node onto the system using the selected electrode segments with the tier system as shown in Fig. 12a. The selected segments ( 6 and 10 for tier 2, 7 and 11 for tier 3, and 8 and 12 for tier 4 ) can be seen in Fig. 4. Note that it is only example of case study using the input dynamic force (e.g. electrodynamic shaker) to the system. Amongst the applications it can be designed as the plucking mechanism so as to create the moderate transient or harmonic responses where the future work will be developed. In Fig. 12b, the parallel and mixed series-parallel electrode segment connections using the quadruple tier are chosen for the analysis. At this case, all four modes (particularly the second and third modes) clearly appear in the power output frequency domain. Again, widening the resonance frequency at the third mode can be achieved up to $15 \mathrm{~Hz}$, just being a slight lower value compared with the results in Fig. 11. Perhaps, this occurs due to the existence of the first twisting mode as being close neighbor to the third mode. However, the power amplitude still shows a reasonably higher value.
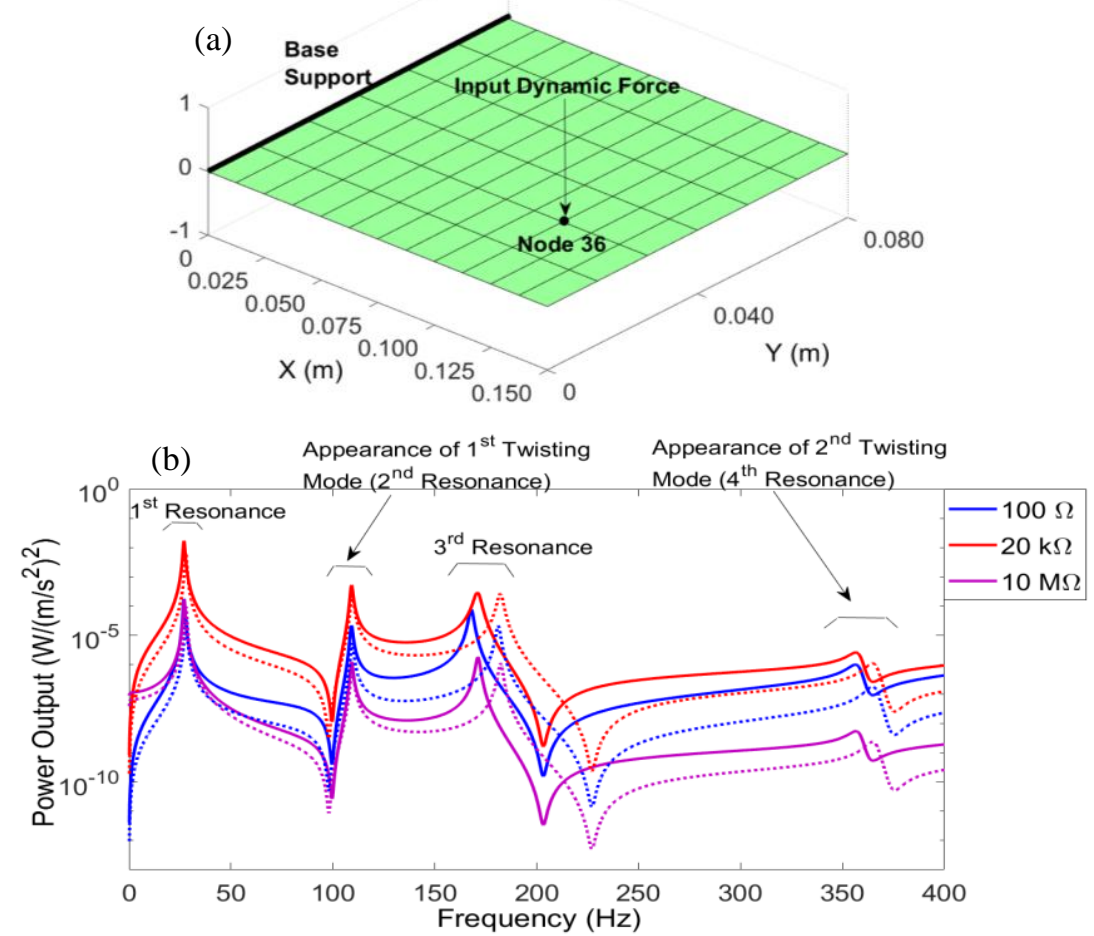

Fig. 12 The system responses : (a) input dynamic force onto smart plate structure and (b) power harvesting FRFs using the quadruple tier network under input dynamic force with the parallel electrode segment connections (solid lines) and mixed series-parallel electrode segment connection (dot lines).

For the time waveform signal responses, the AC to DC signal conversion and charging and discharging processes through the full-wave bridge rectifier and smoothing $\mathrm{RC}$ circuit can be essentially identified from the DC voltage response behaviors. Here, the mixed series-parallel electrode segment was only used for the signal analysis because the previous results showed an effective option to widen the power output with a reasonably higher amplitude. In Figs. 13a-13b, 
the rectifier DC voltage output shows repetitive positive signal responses due to the conductions of the diode pairs (D1 and D2) and (D3 and D4) [33]. As mentioned previously, when the positive half-cycle signal from the piezoelectric system is supplied to the full-wave bridge rectifier, diode pair (D1 and D2) conducts where another diode pair (D3 and D4) switches off. In contrast, when the negative half-cycle signal is supplied to the rectifier, diode pair (D3 and D4) turns to conduct resulting in another diode pair (D1 and D2) to be off. Since the rectifier DC voltage shows a pure ripple signal (very high ripple output as being similar form to that of the rectifier voltage), the smoothing RC circuit system becomes important to reduce the rectifier ripple signal. However, the capacitor ripple voltage can still occur at lower or higher level, depending upon the chosen capacitor and load resistance values. Example of this response behavior can be seen in Fig. 13a where the capacitor ripple voltage with the load resistance of $0.12 \mathrm{M}$ shows lower level compared with the use of $20 \mathrm{k} \Omega$. Again, the fixed capacitance value is $0.1 \mu \mathrm{F}$. Note that lower ripple voltage level can be benefit for quicker charging process compared with higher ripple that can cause heating to the capacitor. To avoid higher ripple, the exponential decay of capacitor ripple voltage level should be less pronounced so as to produce very smooth DC signal. Again, this behavior can be seen from the comparison between the two capacitor ripple voltages with $20 \mathrm{k} \Omega$ and $0.12 \mathrm{M} \Omega$.

Moreover, the capacitor DC current signal can be achieved by the time of charging. The current signal will drop approaching to zero during discharging process where the exponential decay of the capacitor voltage will also occur during that process. It can be seen that capacitor DC current using $20 \mathrm{k} \Omega$ has longer charging time process compared with the use of $0.12 \mathrm{M} \Omega$.

(a)

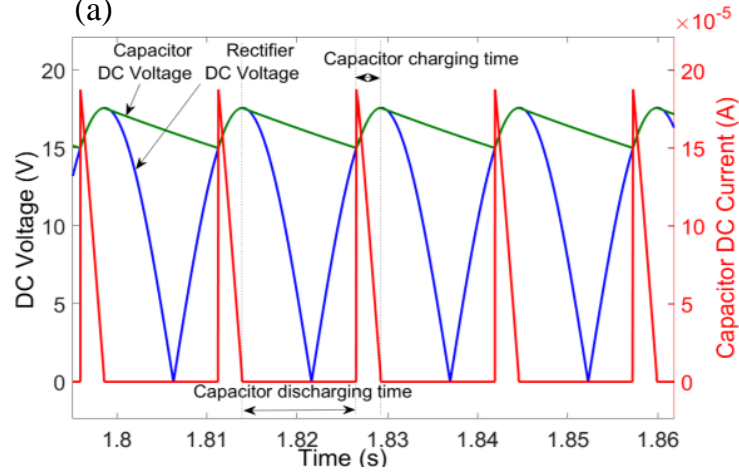

(b)

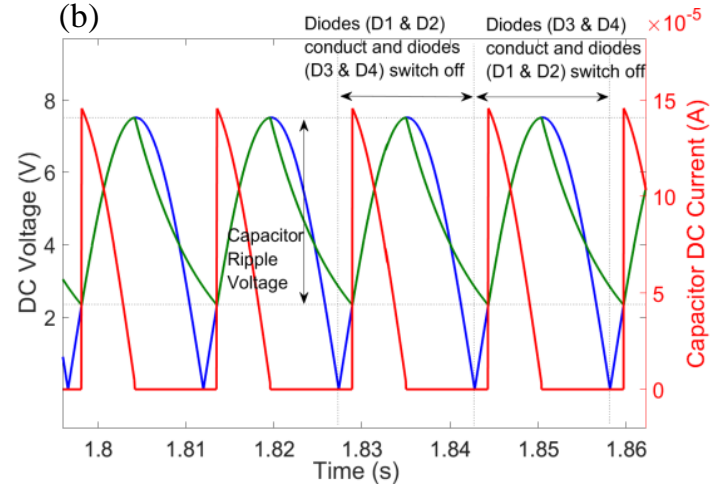

Fig. 13 DC voltage and capacitor DC current time waveform signals from the mixed series-parallel electrode segment connection using the quadruple tier network with the fixed capacitance $C_{d}=0.1 \mu \mathrm{F}$ : (a) $R_{d}=0.12 \mathrm{M} \Omega$ and (b) $R_{d}=20 \mathrm{k} \Omega$
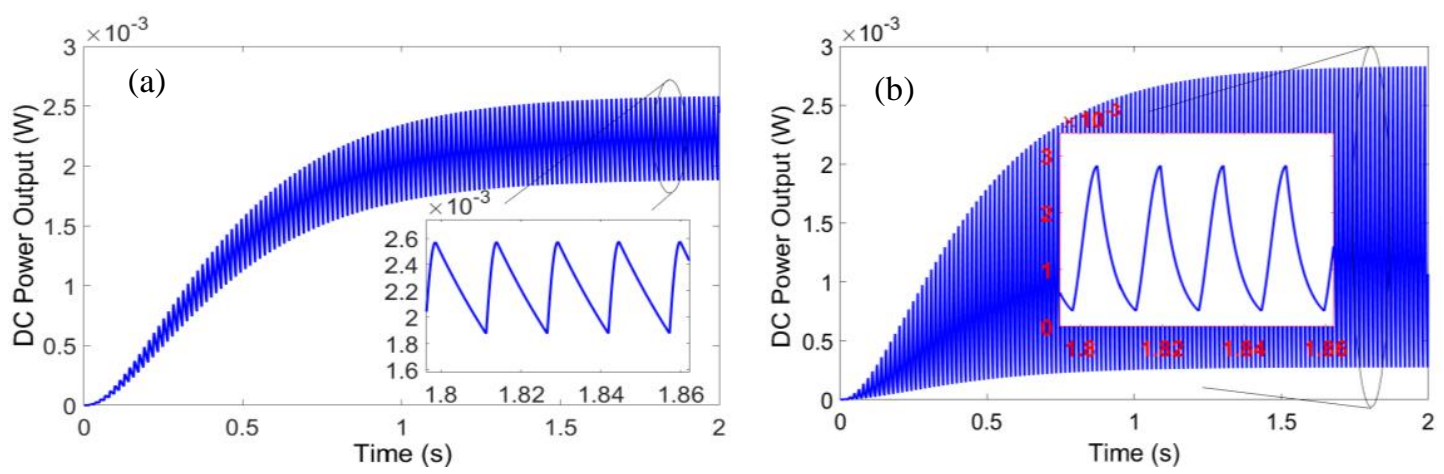

Fig. 14 DC power harvesting across time waveform signals from the mixed series-parallel electrode segment connection using the quadruple tier network with the fixed capacitance $C_{d}=0.1 \mu \mathrm{F}:$ (a) $R_{d}=0.12 \mathrm{M} \Omega$, and (b) $R_{d}=20 \mathrm{k} \Omega$.

However, it can give higher ripple voltage level. Therefore, the use of $0.12 \mathrm{M} \Omega$, as example of this case, can be very effective for faster charging process with lower ripple level. By viewing the DC power across load resistances of $20 \mathrm{k} \Omega$ and $0.12 \mathrm{M} \Omega$ in Figs. $14 \mathrm{a}-14 \mathrm{~b}$, the ripple level still affects the signal outputs. Although the DC power signals approach to similar amplitude, the DC power output signal with $20 \mathrm{k} \Omega$ shows higher fluctuation level compared with the use of $0.12 \mathrm{M} \Omega$.

\section{Conclusion}

This paper has presented the multiple smart plate power harvesters with various circuit connection networks and standard AC-DC circuit interface using the electromechanical finite element dynamic analysis. The functional energy forms of the elemental smart plate structure system were 
essentially developed using the extended Lagrange equations so as to formulate the local element matrices of the electromechanical dynamic equations. The reduced equations into the orthonormalized global matrices have been further developed into the orthonormalized scalar form equations using the electromechanical discretizations associated with the circuit connection patterns. The reduced constitutive numerical models have been used for formulating the smart plate system with the options of either the multiple piezoelectric or electrode segments. The segmented systems with the on-off switching techniques consisting of the parallel, series, and mixed series-parallel piezoelectric/electrode segment connection networks have been further used for formulating the electromechanical multi-mode FRFs and time signal waveforms. The multiple electrode segment connection system was chosen as an example, for analyzing the parametric numerical case studies because it provides the physical insights of the electromechanical system response behaviors and practical implementations.

There are also numerical findings with the proposed techniques addressing six important points:

1. The on-off switching techniques with the tier networks have been used for the numerical analysis of the parallel, series, and mixed series-parallel electrode segment connections. Again, it was mentioned previously that each tier consists of the multiple electrode segments. It was found that increasing tier numbers using the electrode segment connections can affect the power amplitude and frequency domains. The power output for the parallel electrode segments increases with increasing the tier numbers. In the opposite behavior, the power output using series electrode segments shows the reduction of amplitudes. Moreover, the use of the quadruple tier for the parallel and mixed series-parallel electrode segment connection can give higher optimal amplitudes. However, the mixed series-parallel electrode segment connection can be an effective option for widening the resonance frequency when the system is particularly away from the ambient frequency. Therefore, matching the two different frequency environments are essentially required.

2. The plate structure system under input base excitation also gives the effect to the power output frequency analysis due to the strain modes shapes. The second and fourth resonance frequencies disappear due to the second and fourth strain mode shapes (the first and second twisting strain modes, respectively). The major reduction of the power outputs at the twisting modes are due to the tendency of equal strain distribution changes and the input base excitation for the cantilevered plate structure (predominantly creating the transverse modes).

3. With the plate structure system under input dynamic force at particular node as shown, the power outputs for all four modes appear (particularly the second and third modes).

4. For the time waveform signals from the capacitor DC voltage, the reduction of the ripple signal can be achieved using the smoothing RC circuit system depending upon the chosen capacitor and load resistance values. To achieve quicker charging process, higher ripple that can cause heating the capacitor, must be avoided by reducing the exponential decay of capacitor ripple voltage level. The reason is that discharging process of the capacitor DC current signal will also be quicker and charging process can be effectively conducted. Also the DC power across load resistance can also be benefit when the ripple level is very low.

5. The option for using parallel or mixed series-parallel electrode segment connections essentially depends on the applications. In adaptive vibration harvesting cases, the required switching techniques to the particular tiers can be benefit to adjust the power harvesting frequency and amplitude domains.

6. The power output from the smart structure system can be adaptively controlled from higher to lower amplitude levels using the on-off switching tier systems when the ambient vibration environment applied to the system unexpectedly excite high amplitude. This can affect the system potentially having the cycle fatigue due to the repetitive high input excitation and also having brittle nature of the piezoelectric material itself. Conversely, the system response can be optimized using the increase of tier numbers when the input excitation gives a slight lower amplitude.

\section{References}

1. Tzou, H.S., Tseng, C.I.: Distributed vibration control and identification of coupled elastic/piezoelectric systems: finite element formulation and applications, Mech. Syst. Signal Process. 5, 215-231 (1991) 
2. Saravanos, D.A., Heyliger, P.R., Hopkins, D.A.: Layerwise mechanics and finite element for the dynamics analysis of piezoelectric composite plate, Int. J. Solids Struct. 34, 359-378 (1996)

3. Lam, K.Y., Peng, X.Q., Liu, G.R., Reddy, J.N.: A finite element model for piezoelectric composite laminates, Smart Mater. Struct. 6, 583-591 (1997)

4. Detwiler, D.T., Shen, H.M-H., Venkayya, V.B.: Finite element analysis of laminated composite structures containing distributed piezoelectric actuators and sensors, Fin. Elem. Anal. Des. 20, 87-100 (1995)

5. Krommer, M., Irschik, H.: A Reissner-Mindlin type plate theory including the direct piezoelectric and the pyroelectric effect. Acta Mech. 141, 51-69 (2000)

6. Ray, M.C., Sachade, H.M., Finite element analysis of smart functionally graded plates, Int. J. Solids Struct. 43(18-19), 5468-5484 (2006)

7. Krommer, M.: On the correction of the Bernoulli-Euler beam theory for smart piezoelectric beams, Smart Mater. Struct. 10, 668-680 (2001)

8. Krommer, M., Irschik, H.: An electromechanically coupled theory for piezoelastic beams taking into account the charge equation of electrostatics, Acta Mech. 154, 141-158 (2002)

9. Fernandes, A., Pouget, J.: Analytical and numerical approach to piezoelectric bimorph, Int. J. Solids Struct. 40, 4331-4352 (2003)

10. Moita, J.M., Correia, I.F.P., Soares, C.M M.: Active control of adaptive laminated structures with bounded piezoelectric sensors and actuators Comput. Struct. 82(17-19) 1349-1358 (2004).

11. Wang, S.Y.: A finite element model for the static and dynamic analysis of a piezoelectric bimorph, Int. J. Solids Struct. 41, 4075-4096 (2004)

12. Deü, J-F., Benjeddou, A.: Free-vibration analysis of laminated plates with embedded shearmode piezoceramic layers, Int. J. Solids Struct. 42 (7), 2059-2088 (2005)

13. Irschik, H., Krommer, M., Belyaev, A.K., Schlacher, A.K.: Shaping of piezoelectric sensors/actuators for vibrations of slender beams: coupled theory and inappropriate shape functions. J. Intell. Mater. Syst. Struct. 9, 546-554 (1998)

14. Maurini, C., Dell'Isola, F., Del Vescovo., D.: Comparison of piezoelectric networks acting as distributed vibration absorbers, Mech. Syst. Signal Process. 18 (5), 1243-1271 (2004)

15. Thomas, O., Deü, J-F., Ducarne, J.: Vibrations of an elastic structure with shunted piezoelectric patches: efficient finite element formulation and electromechanical coupling coefficients, Int. J. Numer. Methods Eng. 8, 235-268 (2009)

16. Vasques, C.M.A.: Improved passive shunt vibration control of smart piezo-elastic beams using modal piezoelectric transducers with shaped electrodes, Smart Mater. Struct. 21 125003 (2012)

17. Schoeftner, J., Krommer, M.: Single point vibration control for a passive piezoelectric Bernoulli-Euler beam subjected to spatially varying harmonic loads, Acta Mech. 223, 19831998 (2012)

18. Lossouarn, B., Deü, J-F., Aucejo, M., Cunefare, K.A.: Multimodal vibration damping of a plate by piezoelectric coupling to its analogous electrical network, Smart Mater. Struct. 25, 115042 (2016)

19. Roundy, S., Wright, P.K: A piezoelectric vibration based generator for wireless electronics. Smart Mater. Struct., 18, 1131-1142 (2004)

20. Lefeuvre E, Badel A, Richard C, Petit L, Guyomar D.: A comparison between several vibration-powered piezoelectric generators for standalone systems. Sens Actuat A-Phys. 126 405-416 (2006)

21. Shu, Y.C., Lien, I.C.: Analysis of power outputs for piezoelectric energy harvesting systems. Smart Mater. Struct. 15,1499-1512 (2006)

22. Shu, Y.C., Lien, I.C., Wu, W.J.: An improved analysis of the SSHI interface in piezoelectric energy harvesting. Smart Mater. Struct. 16, 2253-2264 (2007)

23. Lallart M, Guyomar D.: An optimized self-powered switching circuit for nonlinear energy harvesting with low voltage output. Smart Mater Struct 17, 035030 (2008)

24. Kim, M., Hoegen, M., Dugundji, J., Wardle, B.L.: Modeling and experimental verification of proof mass effects on vibration energy harvester performance. Smart Mater. Struct. 19, 045023 (2010)

25. Goldschmidtboeing, F., Woias, P.: Characterization of different beam shapes for piezoelectric energy harvesting, J. Micromech. Microeng. 18 ,104013 (2008)

26. Dalzell, $\mathrm{P}$ and Bonello, P.:Analysis of an energy harvesting piezoelectric beam with energy storage circuit. Smart Mater.Struct. 21, 105029 (2012) 
27. Erturk, A.: Assumed-modes modeling of piezoelectric energy harvesters: Euler-Bernoulli, Rayleigh, and Timoshenko models with axial deformations. Comp.Struct. 106-107, 214-227 (2012)

28. Wang, H., Meng, Q.: Analytical modeling and experimental verification of vibration-based piezoelectric bimorph beam with a tip-mass for power harvesting. Mech. Syst. Signal Process. 36,193-209 (2013)

29. Lumentut, M.F., Howard, I.M.: Analytical and experimental comparisons of electromechanical vibration response of a piezoelectric bimorph beam for power harvesting. Mech. Syst. Signal Process. 36, 66-86 (2013)

30. Adhikari, S., Friswell, M.I., Inman, D.J.: Piezoelectric energy harvesting from broadband random vibrations. Smart Mater. Struct. 18115005 (2009)

31 Lumentut, M.F., Howard, I.M.: Parametric design-based modal damped vibrational piezoelectric energy harvesters with arbitrary proof mass offset: Numerical and analytical validations. Mech. Syst. Signal Proc. 68, 562-586 (2016)

32 Tang, L., Wang, J.: Size effect of tip mass on performance of cantilevered piezoelectric energy harvester with a dynamic magnifier, Acta Mech. 228 (11). 3997-4015 (2017)

33. Lumentut, M.F., Howard, I.M.: Intrinsic electromechanical dynamic equations for piezoelectric power harvesters, Acta Mech. 228 (2), 631-650 (2017)

34. Wickenheiser, A.M.: Eigensolution of piezoelectric energy harvesters with geometric discontinuities: Analytical modelling and validation, J. Intell. Mater. Syst. Struct. 24, 729744 (2013)

35. Zhou, S., Hobeck, J.D., Cao, J., Inman, D.J.: Analytical and experimental investigation of flexible longitudinal zigzag structures for enhanced multi-directional energy harvesting, Smart Mater. Struct. 26, 035008 (2017)

36. Lumentut, M.F., Howard, I.M.: Effect of shunted piezoelectric control for tuning piezoelectric power harvesting system responses - analytical techniques, Smart Mater. Struct. 24, 105029 (2015)

37. Lumentut, M.F., Howard, I.M.: Electromechanical analysis of an adaptive piezoelectric energy harvester controlled by two segmented electrodes with shunt circuit networks, Acta Mech. 228(4), 1321-1341 (2017)

38. Lumentut, M.F., Francis, L.A., Howard, I.M.: Analytical techniques for broadband multielectromechanical piezoelectric bimorph beams with multifrequency power harvesting, IEEE Trans. Ultrason. Ferroelectr. Freq. Control. 59, 1555-1568 (2012)

39. Zhang, H., Afzalul, K.: Design and analysis of a connected broadband multi-piezoelectricbimorph-beam energy harvester, IEEE Trans. Ultrason. Ferroelectr. Freq. Control. 61, 1016$1023(2014)$

40. Xiong, X., Oyadiji, S.O.: Modal optimization of doubly clamped base-excited multilayer broadband vibration energy harvesters, J. Intel. Mat. Syst. Struct. 26(16). 2216-2241 (2014)

41. Lien., I.C., Shu, Y.C.: Array of piezoelectric energy harvesting by the equivalent impedance approach, Smart Mater. Struct. 21, 082001 (2012)

42. Lin, H.C., Wu, P.H., Lien, I.C., Shu, Y.C.: Analysis of an array of piezoelectric energy harvesters connected in series. Smart Mater. Struct. 22, 094026 (2013)

43. Wu, P.H., Shu, Y.C.: Finite element modeling of electrically rectified piezoelectric energy harvesters. Smart Mater. Struct. 24, 094008 (2015)

44. Aridogan, U., Basdogan, I., Erturk, A.: Multiple patch-based broadband piezoelectric energy harvesting on plate-based structures, J. Intel. Mat. Syst. Struct 25(14), 1664-1680 (2014)

45. Bayik, B., Aghakhani, A., Basdogan, I., Erturk, A: Equivalent circuit modeling of a piezopatch energy harvester on a thin plate with AC-DC conversion, Smart Mater. Struct. 25, 055015 (2016)

46. Yoon, H., Youn, B.D., Kim, H.S. Kirchhoff plate theory-based electromechanically-coupled analytical model considering inertia and stiffness effects of a surface-bonded piezoelectric patch, Smart Mater. Struct. 25, 025017 (2016)

47. Samanta, B., Ray, M.C., Bhatacharya, R.: Finite element model for active control of intelligent structures, AIAA J. 34(9), 1885-1893 (1996)

48. Liu, G.R, Dai, K.Y., Lim, K.M.: Static and vibration control composite laminates integrated with piezoelectric sensors and actuators using the radial point interpolation method, Smart Mater. Struct. 13, 1438-1447 (2004)

49. Kapuria, S., Yaqoob Yasin, M.: Active vibration control of smart plates using directional actuation and sensing capability of piezoelectric composites, Acta Mech. 224, 1185-1199 (2013) 
50. Venkata Rao K., Raja, S., Munikenche Gowda, T.: Finite element modelling and vibration control study of active plate with debonded piezoelectric actuators, Acta Mech 2252923 2942 (2014)

51. Elvin, N.G., Elvin, A.A., Coupled finite element-circuit simulation model for analyzing piezoelectric energy generators, J. Intell. Mater. Syst. Struct. 20, 587-595 (2009)

52. Friswell, M.I., Adhikari, S.: Sensor shape design for piezoelectric cantilever beams to harvest vibration energy. J. App. Phy. 108, 014901 (2010)

53. DeMarqui, J Jr., Erturk, A., Inman, D.J.: An electromechanical finite element model for piezoelectric energy harvester plates, J. Sound Vib. 327(1-2), 9-25 (2009)

54. Aladwani, A., Arafa, M., Aldraihem, O., Baz, A.: Cantilevered piezoelectric energy harvester with a dynamic magnifier, J. Vib. Acoust. 134031004 (2012)

55. Lumentut, M.F., Howard, I.M.: Electromechanical finite element modelling for dynamic analysis of a cantilevered piezoelectric energy harvester with tip mass offset under base excitations, Smart Mater. Struct., 23 (9), 095037 (2014)

56. Tiersten, H.F., Linear piezoelectric plate vibrations (New York: Plenum, 1969)

57. Nye, J.F.: Physical Properties of Crystals: Their representation by tensors and matrices (Oxford: Clarendon, 1984)

58. Ikeda T 1990 Fundamentals of piezoelectricity (New York: Oxford University Press)

59. Lumentut, M.F.: Mathematical dynamics of electromechanical piezoelectric energy harvester $\mathrm{PhD}$ Thesis Curtin University (Australia, 2011) http://espace.library.curtin.edu. $\mathrm{au} / \mathrm{R} /$ ?func=dbin-jump-full\&object_id=186549\&local_base=gen01-era02

60. Kirchhoff, G.R.: Über das Gleichgewicht und die Bewegung einer elastischen Scheibe J. Reine Angew. Math. 40, 51-88 (1850)

61. Sokolnikoff, I.S.: Mathematical theory of elasticity ( $2^{\text {nd }}$ Edition McGraw-Hill, 1956)

62. Adini, A., Clough, R.W.: Analysis of plate bending by the finite element method, Report submitted to the National Science Foundation, G7337, 1960

63. Melosh, R.J.: Basis for derivation of matrices for the direct stiffness method, AIAA J. 1, 1631-1637 (1963).

64. Petyt, M.: Introduction to finite element vibration analysis ( $2^{\text {nd }}$ Edition Cambridge University Press, 2010)

65. Ottman, G.K, Hofmann, H.F., Bhatt, A.C., Lesieutre, G.A.: Adaptive Piezoelectric Energy Harvesting Circuit for Wireless Remote Power Supply, IEEE Trans. Power Elec., 17(5), 669$676(2002)$

66. http://www.efunda.com/materials/piezo/material_data/matdata_output.cfm?Material_ID=PZT$5 \mathrm{~A}$ 